\title{
Rigidity of a non-elliptic differential inclusion related to the Aviles-Giga conjecture
}

\author{
Xavier Lamy* $\quad$ Andrew Lorent ${ }^{\dagger} \quad$ Guanying Peng ${ }^{\ddagger}$
}

\begin{abstract}
In this paper we prove sharp regularity for a differential inclusion into a set $K \subset \mathbb{R}^{2 \times 2}$ that arises in connection with the Aviles-Giga functional. The set $K$ is not elliptic, and in that sense our main result goes beyond Šverák's regularity theorem on elliptic differential inclusions. It can also be reformulated as a sharp regularity result for a critical nonlinear Beltrami equation. In terms of the Aviles-Giga energy, our main result implies that zero energy states coincide (modulo a canonical transformation) with solutions of the differential inclusion into $K$. This opens new perspectives towards understanding energy concentration properties for Aviles-Giga: quantitative estimates for the stability of zero energy states can now be approached from the point of view of stability estimates for differential inclusions. All these reformulations of our results are strong improvements of a recent work by the last two authors Lorent and Peng, where the link between the differential inclusion into $K$ and the Aviles-Giga functional was first observed and used. Our proof relies moreover on new observations concerning the algebraic structure of entropies.
\end{abstract}

\section{Introduction}

The Aviles-Giga functional for $u \in W^{2,2}(\Omega)$ over a bounded domain $\Omega \subset \mathbb{R}^{2}$ is given by

$$
I_{\varepsilon}(u)=\int_{\Omega}\left(\varepsilon\left|\nabla^{2} u\right|^{2}+\frac{\left(1-|\nabla u|^{2}\right)^{2}}{\varepsilon}\right) d x .
$$

Here $\nabla^{2} u$ is the Hessian matrix of the scalar-valued function $u$ and $\varepsilon>0$ is a small parameter. This is a second order functional that (subject to appropriate boundary conditions) models phenomena from thin film blistering to smectic liquid crystals, and is also the most natural higher order generalization of the Cahn-Hilliard functional. The Aviles-Giga conjecture for the $\Gamma$-limit of $I_{\varepsilon}$ is one of the central conjectures in the theory of $\Gamma$-convergence and has attracted a great deal of attention; see for example AG87, AG96, ADLM99, DMKO01, DLO03. One of the main theorems in the theory of the AvilesGiga functional is the characterization of "zero energy states" of the functional by Jabin, Otto and Perthame [JOP02]. A zero energy state is a function $u$ that is a strong limit of a sequence $u_{\varepsilon}$ with $I_{\varepsilon}\left(u_{\varepsilon}\right) \rightarrow 0$ as $\varepsilon \rightarrow 0$. Clearly $u$ satisfies the Eikonal equation given by

$$
|\nabla u|=1 \quad \text { a.e. }
$$

A formulation of the Jabin-Otto-Perthame theorem involves the notion of entropies, which is a central tool for the analysis of the Aviles-Giga functional. Non-technically speaking, entropies are smooth

*Institut de Mathématiques de Toulouse, UMR 5219, Université de Toulouse, CNRS, UPS IMT, F-31062 Toulouse Cedex 9, France. Email: Xavier.Lamy@math.univ-toulouse.fr

${ }^{\dagger}$ Department of Mathematical Sciences, University of Cincinnati, Cincinnati, OH 45221, USA. Email: lorentaw@uc.edu

${ }^{\ddagger}$ Department of Mathematics, University of Arizona, Tucson, AZ 85721, USA. Email: gypeng@math.arizona.edu 
vector fields $\Phi: \mathbb{R}^{2} \rightarrow \mathbb{R}^{2}$ such that $\operatorname{div} \Phi\left(\nabla^{\perp} u\right)=0$ if $u$ is a smooth solution to the Eikonal equation. For weak solutions $u=\lim _{\varepsilon \rightarrow 0} u_{\varepsilon}$ with $\sup _{\varepsilon} I_{\varepsilon}\left(u_{\varepsilon}\right)<\infty, \operatorname{div} \Phi\left(\nabla^{\perp} u\right)$ are measures, called entropy measures, that detect the jump in $\nabla u$ (see (13) and (39) for a detailed definition of entropies). The Jabin-Otto-Perthame theorem states that if $u$ is a solution to the Eikonal equation and if for every entropy $\Phi$ the function $u$ satisfies $\operatorname{div} \Phi\left(\nabla^{\perp} u\right)=0$ distributionally in $\Omega$, then $\nabla u$ is smooth outside a locally finite set. Indeed in any convex neighborhood $U$ of a singular point $x_{0}$ the vector field $\nabla^{\perp} u$ forms a vortex around $x_{0}$ in $U$.

Recently the second two authors provided a generalization of this result in [LP18: the same conclusion holds under the weaker assumption that $m=\nabla^{\perp} u$ satisfies

$$
\operatorname{div} \Sigma_{j}(m)=0 \text { distributionally in } \Omega \text { for } j=1,2 \text {, }
$$

where $\Sigma_{1}, \Sigma_{2} \in C^{\infty}\left(\mathbb{R}^{2} ; \mathbb{R}^{2}\right)$ are the entropies introduced by Jin and Kohn JK00. (see (4) in Section 1.1 below) and further used by Ambrosio, De Lellis and Mantegazza [ADLM99] to formulate a $\Gamma$ limit conjecture for the Aviles-Giga functional. A necessary condition for their conjecture to hold is that the Jin-Kohn entropy productions $\operatorname{div} \Sigma_{j}\left(\nabla^{\perp} u\right)$, if they are measures, control all other entropy productions $\operatorname{div} \Phi\left(\nabla^{\perp} u\right)$. Hence the main result in [LP18] shows this in the particular case when the Jin-Kohn entropy productions are zero. A key new perspective in LP18 is to associate to a function $u$ satisfying $\operatorname{div} \Sigma_{j}\left(\nabla^{\perp} u\right)=0$ and $|\nabla u|=1$ a mapping $F: \Omega \rightarrow \mathbb{R}^{2}$ that satisfies a differential inclusion into a set $K$ (see (7)) determined by the two Jin-Kohn entropies $\Sigma_{j}$. The main result in [LP18, shows regularity for any $F$ satisfying the differential inclusion $D F \in K$, provided $F$ was originally associated to a function $u$ as above. This is the case if $F$ already has some regularity, e.g. $F \in W^{2,1}$ LP18, Theorem 5]. Our aim in the present work is to prove a more natural regularity result for the differential inclusion $D F \in K$ (regardless of whether $F$ was originally associated to a function $u$ ), removing this extra regularity assumption.

Note that the Eikonal equation (11) can be equivalently formulated as

$$
|m|=1 \text { a.e., } \quad \operatorname{div} m=0
$$

by identifying $m=\nabla^{\perp} u$. In this setting the main result of [LP18] shows regularity of $m$ satisfying (3) and (2). There is a correspondence between Lipschitz maps $F$ satisfying the above-mentioned differential inclusion $D F \in K$ a.e., and unit vector fields $m$ satisfying (2) but not necessarily divergence free. Hence proving a natural regularity result for the differential inclusion into $K$ amounts to generalizing the main result in [LP18 by removing the assumption that div $m=0$. This is one of the formulations of our main results: if a vector field $m: \Omega \rightarrow \mathbb{S}^{1}$ satisfies (2), then once again the regularity and rigidity of zero energy states are valid (see Theorem 1).

Formulated in terms of differential inclusions (see Theorem 2), this constitutes a sharp regularity result for the differential inclusion into $K$, compared to the corresponding one in LP18. The set $K$ is not elliptic in the sense of Šverák [Š93] and DiPerna DiP85]. As such our Theorem 2 is (to our knowledge) the first regularity/rigidity result for non-elliptic differential inclusions and opens the possibility of regularity results for differential inclusions under much more general hypotheses than those of $[\check{S} 93$.

However our principal aim is the study of the Aviles-Giga conjecture. As will be explained, we envision our main result as a technical tool in the study of the energy concentration. Specifically we are interested to attack this problem by establishing quantitative stability estimates for the differential inclusion into $K$. The first step in such a program is to establish rigidity of the differential inclusion into $K$ itself, and is the purpose of the present work. 


\subsection{Statement of the main results}

To state our main result, let us first introduce the Jin-Kohn entropies $\Sigma_{1}, \Sigma_{2} \in C^{\infty}\left(\mathbb{R}^{2} ; \mathbb{R}^{2}\right)$. For $v \in \mathbb{R}^{2}$, the two entropies are given by

$$
\begin{aligned}
& \Sigma_{1}(v)=\left(v_{2}\left(1-v_{1}^{2}-\frac{v_{2}^{2}}{3}\right), v_{1}\left(1-v_{2}^{2}-\frac{v_{1}^{2}}{3}\right)\right), \\
& \Sigma_{2}(v)=\left(-v_{1}\left(1-\frac{2 v_{1}^{2}}{3}\right), v_{2}\left(1-\frac{2 v_{2}^{2}}{3}\right)\right) .
\end{aligned}
$$

Note that $\Sigma_{2}(v)=R_{\frac{\pi}{4}} \Sigma_{1}\left(R_{-\frac{\pi}{4}} v\right)$, where $R_{\theta}$ denotes the rotation of angle $\theta$. As mentioned earlier, our main result can be stated either in terms of unit vector fields $m$ that are not necessarily divergence free, or in terms of differential inclusions (see also Theorem 5 below in terms of nonlinear Beltrami equations). We first adopt the unit vector field point of view:

Theorem 1. Let $\Omega \subset \mathbb{R}^{2}$ be an open set and $m: \Omega \rightarrow \mathbb{R}^{2}$ satisfy

$$
|m|=1 \quad \text { a.e., } \quad \operatorname{div} \Sigma_{j}(m)=0 \quad \text { in } \mathcal{D}^{\prime}(\Omega) \quad \text { for } j=1,2 .
$$

Then $m$ is locally Lipschitz outside a locally finite set of points $S$. Moreover, for any singular point $\zeta \in S$ there exists $\alpha \in\{ \pm 1\}$ such that in any convex neighborhood $\mathcal{O} \subset \subset \Omega$ of $\zeta$,

$$
m(x)=\alpha i \frac{x-\zeta}{|x-\zeta|} \quad \text { for all } x \in \mathcal{O},
$$

where $i \in \mathbb{C}$ is identified with the counterclockwise rotation of angle $\frac{\pi}{2}$ in $\mathbb{R}^{2}$.

As mentioned earlier, this result is a strong extension of the main result in [LP18, which states the same regularity for $m$ satisfying the additional divergence free assumption, i.e., $m$ satisfying the Eikonal equation, and it opens new perspectives towards energy concentration results for Aviles-Giga minimizers.

To give the equivalent statement in terms of differential inclusions, we introduce the mapping $P:[0,2 \pi) \rightarrow \mathbb{R}^{2 \times 2}$ given by

$$
\begin{aligned}
P(\theta) & :=\left(\begin{array}{c}
i \Sigma_{1}\left(e^{i \theta}\right) \\
i \Sigma_{2}\left(e^{i \theta}\right)
\end{array}\right) \\
& =\left(\begin{array}{cc}
-\frac{2}{3} \cos ^{3}(\theta) & \frac{2}{3} \sin ^{3}(\theta) \\
-\sin (\theta)\left(1-\frac{2}{3} \sin ^{2}(\theta)\right) & -\cos (\theta)\left(1-\frac{2}{3} \cos ^{2}(\theta)\right)
\end{array}\right),
\end{aligned}
$$

and we define

$$
K:=\{P(\theta): \theta \in[0,2 \pi)\} \subset \mathbb{R}^{2 \times 2} .
$$

Theorem 2. Let $\Omega \subset \mathbb{R}^{2}$ be an open set, and $F: \Omega \rightarrow \mathbb{R}^{2}$ be a Lipschitz map satisfying the differential inclusion $D F \in K$ a.e. Then DF is locally Lipschitz outside a locally finite set of points $S$. Moreover, for any singular point $\zeta \in S$ there exists $\alpha \in\{ \pm 1\}$ such that in any convex neighborhood $\mathcal{O} \subset \subset \Omega$ of $\zeta$,

$$
\left(\begin{array}{l}
F_{1,1}(x)+F_{2,2}(x) \\
F_{2,1}(x)-F_{1,2}(x)
\end{array}\right)=\alpha i \frac{x-\zeta}{|x-\zeta|} \quad \text { for all } x \in \mathcal{O} .
$$

As already explained, Theorem 2 is a considerable improvement on the corresponding result in [LP18, where the same rigidity was proved under the additional - and unnatural - assumption $F \in$ $W^{2,1}(\Omega)$. Our Theorem 2, in contrast, is a sharp regularity result. In the following, we also reformulate this result as sharp regularity for a nonlinear Beltrami equation; see Theorem 5 below.

Remark 3. Since Theorems 1 and 2 are local statements, in the sequel we will assume without loss of generality that $\Omega$ is smooth, bounded and simply connected. 


\subsection{Differential inclusions}

Regularity of differential inclusions is a classical subject. Let

$$
C O_{+}(n):=\{\lambda R: R \in S O(n)\} .
$$

The first and best known result is analyticity of the differential inclusion $D u \in C O_{+}(2)$. This differential inclusion is nothing other than the Cauchy-Riemann equations and analyticity is one of the first basic theorems of complex analysis. Rigidity of the differential inclusion $D u \in C O_{+}(3)$ was studied by Liouville in 1850 for $C^{3}$ mappings Lio50. The generalization of this result has been a topic of great interest in the Quasiconformal analysis community Geh62, Res67, BI82, IM93, MvY99. Another classical example is the differential inclusion into the set $\left\{A \in \mathbb{R}^{n \times n}: A^{T}=A, \operatorname{Tr}(A)=0\right\}$, which corresponds to the Laplace equation $\Delta u=0$ in $\mathbb{R}^{n}$.

We say a set $S \subset \mathbb{R}^{m \times n}$ has a Rank-1 connection if there exist $A, B \in S$ with $\operatorname{Rank}(A-B)=1$. It is a simple fact that if a set $S$ has a Rank-1 connection then one can construct wild solutions to the differential inclusion $D w \in S$ through the construction of laminates. It is tempting to conjecture that if a set $S$ has no Rank-1 connections then the differential inclusion $D w \in S$ has higher regularity, but this is completely false. This falsity is a key fact in the recent spectacular progress in counter-examples to regularity of PDE $\mathrm{Mv03}$, Sze04, MRv05. In $\mathbb{R}^{2 \times 2}, \operatorname{Rank}(A-B)=1$ if and only if $\operatorname{det}(A-B)=0$. So for any connected analytic set $S \subset \mathbb{R}^{2 \times 2}$ without Rank-1 connections, by Lojasiewicz inequality (up to the change of sign) there exists some $p \in \mathbb{N}$ such that $\operatorname{det}(A-B) \gtrsim|A-B|^{p}$. For an elliptic set, which is a set whose tangent space at any point does not contain Rank-1 connections, the inequality holds for $p=2$. In $\check{\text { Š93 }}$ Šverák proved

Theorem 4 (Šverák [ัS93]). Let $\Omega \subset \mathbb{R}^{2}$ be open and bounded and $S \subset \mathbb{R}^{2 \times 2}$ be a closed connected smooth submanifold without Rank-1 connections. Further assume that $S$ is elliptic. Then every Lipschitz $w: \Omega \rightarrow \mathbb{R}^{2}$ satisfying $D w \in S$ a.e. is smooth.

Since $\mathrm{CO}_{+}(2)$ is a closed smooth connected elliptic set, this result is a far reaching generalization of analyticity of Lipschitz solutions of the Cauchy-Riemann equations (for more details on this topic see [Lor14a]). To our knowledge this is the most general result on regularity of differential inclusions. The set $K$ defined by (7) is not elliptic and easy examples show that the regularity provided by Theorem 2 is optimal: differential inclusions into $K$ do have singularities, however the nature of the singularities is explicitly given by (8) on any given convex subdomain $\mathcal{O} \subset \subset \Omega$ containing a singularity $\zeta$. Another way to formulate this is as follows. Let $\theta(x)$ be defined by $P(\theta(x))=D F(x)$, then for some $\alpha \in\{1,-1\}$,

$$
e^{i \theta(x)}=\alpha i \frac{x-\zeta}{|x-\zeta|} \quad \text { for all } x \in \mathcal{O},
$$

i.e. $e^{i \theta(x)}$ forms a vortex around $\zeta$.

Theorem 2 can also be formulated as a result for a nonlinear Beltrami equation as done in [LP18. Namely, we also have

Theorem 5. Given an open set $\tilde{\Omega} \subset \mathbb{C}$, let $v: \tilde{\Omega} \rightarrow \mathbb{C}$ be a Lipschitz function that satisfies the nonlinear Beltrami system

$$
\frac{\partial v}{\partial \bar{z}}(z)=\frac{4}{3}\left(\frac{\partial v}{\partial z}(z)\right)^{3}, \quad\left|\frac{\partial v}{\partial z}(z)\right|=\frac{1}{2} \quad \text { for a.e. } z \in \tilde{\Omega},
$$

then $\nabla v$ is locally Lipschitz outside a discrete set $S$, and for any convex set $\mathcal{O} \subset \subset \tilde{\Omega}$, we have $\operatorname{Card}(\mathcal{O} \cap S)=1$.

This result is again a strong improvement of the corresponding result in LP18, where the same regularity for $v$ was established under an additional $W^{2,1}$ regularity assumption on $v$. Equation (9) can 
be recognized as a nonlinear Beltrami system by introducing $\mathcal{H}_{0}(\xi):=\frac{4}{3} \xi^{3}$. Then this equation can be written as $\frac{\partial v}{\partial \bar{z}}(z)=\mathcal{H}_{0}\left(\frac{\partial v}{\partial z}(z)\right),\left|\frac{\partial v}{\partial z}\right|=\frac{1}{2}$ a.e. Equations of the form $\frac{\partial v}{\partial \bar{z}}=\mathcal{H}\left(z, \frac{\partial v}{\partial z}\right)$ have received a great deal of study in the last few years. Under the assumptions that

(i) $z \mapsto \mathcal{H}(z, w)$ is measurable,

(ii) and for $w_{1}, w_{2} \in \mathbb{C},\left|\mathcal{H}\left(z, w_{1}\right)-\mathcal{H}\left(z, w_{2}\right)\right| \leq k\left|w_{1}-w_{2}\right|$ for some $k<1$,

a powerful existence and regularity theory of nonlinear Beltrami equations has been developed; see Boj74, Iwa76, BI76, AIS01, AIM09. Our system (9) corresponds to a critical case, since the mapping $\mathcal{H}_{0}$ has Lipschitz constant 1 on the circle with radius $\frac{1}{2}$. As such Theorem 5 does not follow from any of the known regularity results, and is to our knowledge the first to hold in the critical and genuinely nonlinear case. For linear Beltrami equations there are many powerful results in the critical case, see AIM09, Chapter 20]. Note also that the power 3 nonlinearity $\mathcal{H}_{0}$ appears as an interesting particular case in [ACF ${ }^{+} 19$, Remark 15].

\subsection{The Aviles-Giga functional}

As noted at the beginning the Aviles-Giga functional is a higher order generalization of the CahnHilliard functional. In 1977 Modica-Mortola MM77, proved that the Cahn-Hilliard functional $\Gamma$ converges to the surface area of the jump set of the limiting function. This proved a conjecture of De Giorgi [DGF75] and was one of the first results in $\Gamma$-convergence. Since then vast literature in applying $\Gamma$-convergence to problems in Calculus of Variations and PDE has evolved. One of the main conjectures in the field of $\Gamma$-convergence is, loosely stated, the conjecture that the $\Gamma$-limit of the Aviles-Giga functional is an energy functional of the form

$$
I_{0}(\nabla u)=c \int_{J_{\nabla u}}\left|\nabla u^{+}-\nabla u^{-}\right|^{3} d \mathcal{H}^{1} \quad \text { for } u \text { solving }|\nabla u|=1,
$$

where $J_{\nabla u}$ is a one-dimensional jump set, and $\nabla u^{ \pm}$denote traces of $\nabla u$ on each side of the jump set. The principal reason that makes the Aviles-Giga conjecture much more difficult than the $\Gamma$-convergence of the Cahn-Hilliard functional is that the power 3 scaling of the Aviles-Giga functional makes the BV function theory inapplicable, and it is not even clear that $\nabla u$ has a one-dimensional jump set. If one assumes that $\nabla u$ is $B V$, then the conjecture is settled in ADLM99, Pol07, CDL07. However a strong limit $u$ of a sequence of bounded Aviles-Giga energy does not in general satisfy $\nabla u \in B V$ (see [ADLM99]), and despite considerable efforts from multiple authors [AG87, AG96, ADLM99, DMKO01, DLO03 the $\Gamma$-convergence conjecture remains very much open.

Similar questions and open problems arise in the context of a micromagnetics energy first studied by Rivière and Serfaty [RS01, RS03. There some issues are simplified due to the fact that vortices can not appear in the limit, but the works on both problems have certainly influenced each other (see e.g. the rectifiability results [DLO03, AKLR02]). Analogous issues are also of importance in the study of large deviation principles for some stochastic processes, where the limiting equations are scalar conservation laws BBMN10.

The precise conjecture in [ADLM99] is that the $\Gamma$-limit is (up to a constant) the total mass of the entropy measure

$$
\mu=\left\|\begin{array}{c}
\operatorname{div} \Sigma_{1}\left(\nabla^{\perp} u\right) \\
\operatorname{div} \Sigma_{2}\left(\nabla^{\perp} u\right)
\end{array}\right\|
$$

which is indeed controlled by the energy, and coincides with the cubic jump cost when $\nabla u \in B V$. The main choke point for progress on the conjecture is the lack of methods or tools to show that $\mu$ is concentrated on a rectifiable one-dimensional set. In this direction, De Lellis and Otto prove in DLO03 that the points of positive one-dimensional density for $\mu$ do form an $\mathcal{H}^{1}$-rectifiable set, but so far concentration for $\mu$ remains completely out of reach. It is not even known that $\mu$ is singular with 
respect to the Lebesgue measure. Progress towards such concentration results is in truth our main motivation.

Analogous questions can be studied for weak solutions of Burgers' equation, motivated by similar $\Gamma$-convergence conjectures related to large deviation principles for some stochastic processes; see BBMN10] and the references therein. There one-dimensional concentration of the entropy measure is also open but it is shown in LO18 that the set of non-Lebesgue points has dimension at most one (very recently this has been extended to more general conservation laws in [Mar]). In the Aviles-Giga setting such result is not known yet.

The most natural way to tackle the problem of concentration is to prove a Poincaré type inequality, that would bound the distance of $u$ to zero energy states in terms of the Aviles-Giga energy. This is in the spirit of what was achieved in [LO18] in the context of Burgers' equation, and this was also the motivation for Lor14b. Part of our interest in proving Theorem 2 is to develop a new tool to establish such an inequality in the Aviles-Giga setting. Specifically we are motivated by the recent powerful quantitative stability estimates for the rigidity of differential inclusion into $S O(n), C O_{+}(n)$ [FJM02, FZ05] and have a view to proving a stability estimate for the differential inclusion into $K$.

The first step in proving quantitative stability is to show rigidity for the differential inclusion itself and that is what is achieved in Theorem 2, Our hope is to obtain in a future step a stability estimate of the form

$$
\inf _{\left\{G \in W^{1, \infty}\left(B_{1}\right) \text { s.t. } \nabla G \in K\right\}} \int_{B_{1}}|\nabla F-\nabla G| \lesssim\left(\int_{B_{1}} \operatorname{dist}(\nabla F, K)\right)^{\alpha}
$$

for all Lipschitz $F$ and for some $\alpha>0$. Then the crucial interest of Theorem 1/2 is that it tells us that the states of exact differential inclusion coincide (modulo a canonical transformation) with the zero energy states for Aviles-Giga. Therefore combining (10) with the quantitative Hodge estimate in ADLM99, Theorem 4.3] would imply

$$
\inf _{\{\nabla v \text { zero energy state }\}}|\nabla u-\nabla v| \lesssim \mu\left(B_{1}\right)^{\alpha},
$$

which is the above mentioned Poincaré type inequality.

\subsection{Proof sketch and plan of the article}

Throughout this paper, we use the notation $A \lesssim B$ to indicate $A \leq c B$ for some constant $c$ independent of the underlying domain or functions. Recall that our goal is to show regularity and rigidity of a vector field $m$ that satisfies

$$
|m|=1 \text { a.e., } \quad \text { and } \quad \operatorname{div} \Sigma_{j}(m)=0 \text { for } j=1,2 .
$$

In [LP18] this was achieved under the additional assumption that $\operatorname{div} m=0$. Thus the proof of Theorem 1 will consist in proving that $\operatorname{div} m=0$ so that we can appeal to [LP18] to conclude. An indication that this should be true is the explicit identity

$$
\begin{aligned}
\operatorname{div} m= & -2 m_{1} m_{2} \operatorname{div} \Sigma_{1}(m) \\
& +\left(m_{1}^{2}-m_{2}^{2}\right) \operatorname{div} \Sigma_{2}(m) \quad \text { if } m: \mathbb{R}^{2} \rightarrow \mathbb{S}^{1} \text { is smooth. }
\end{aligned}
$$

If $m$ is not regular enough to apply the chain rule, this identity can not be computed. It is then natural to try approximating $m$ with a mollification $m_{\varepsilon}=m * \rho_{\varepsilon}$. But $m_{\varepsilon}$ does not take values into $\mathbb{S}^{1}$, and a lot of additional terms appear in the identity (12). These terms involve the lack of " $\mathbb{S}^{1}$-valuedness" through the nonlinear commutator

$$
1-\left|m_{\varepsilon}\right|^{2}=[\Pi(m)]_{\varepsilon}-\Pi\left(m_{\varepsilon}\right), \quad \Pi=|\cdot|^{2} .
$$


It was remarked in DLI15] that if $m$ is " $\frac{1}{3}$-differentiable" in a strong enough sense $\left(W^{\frac{1}{3}, 3}\right.$ in that case), then commutator estimates (introduced in [CET94 in the context of Euler's equation) imply that such additional terms vanish in the limit $\varepsilon \rightarrow 0$. Here some regularity of $m$ is available thanks to [LP18, where it is shown that any $m$ satisfying (11) has the Besov regularity $B_{4, \infty}^{\frac{1}{3}}$. (This is related to a weak coercivity property of the differential inclusion into $K$, namely $\operatorname{det}(A-B) \gtrsim|A-B|^{4}$ for all $A, B \in K$, and to standard compensated compactness tools for estimating determinants. See [FK12] for a regularity result in a similar spirit.) This Besov regularity does not imply the $W^{\frac{1}{3}, 3}$ regularity used in DLI15; it is not good enough to ensure that the commutator terms tend to zero, and to obtain (12) for our map $m$. It is however good enough to bound the commutator terms in order to deduce that

$$
\operatorname{div} m \in L_{\mathrm{loc}}^{\frac{4}{3}}
$$

and this constitutes the first step of our proof in Section 2. In the same spirit, throughout the article we make extensive use of commutator estimates to derive useful information from identities that are valid for smooth $\mathbb{S}^{1}$-valued maps.

The rest of the proof is to obtain $\operatorname{div} m=0$ from this preliminary estimate. To that end we use a tool already crucial in [DMKO01, JOP02, IM12, DLI15, LP18, GL20, namely entropies and entropy productions. The terminology comes from an analogy with scalar conservation laws, where similar objects play an important role. An entropy is a $C^{2} \operatorname{map} \Phi: \mathbb{S}^{1} \rightarrow \mathbb{R}^{2}$ that provides an admissible renormalization of the Eikonal equation (3) in the sense (similar to [DL89] for transport equations) that $\operatorname{div} \Phi(m)=0$ for all smooth solutions of the Eikonal equation. Applying the chain rule one sees that this is equivalent to the requirement

$$
e^{i t} \cdot \frac{d}{d t} \Phi\left(e^{i t}\right)=0
$$

If a solution $m$ is $B V$, one can still apply the chain rule and see that $\operatorname{div} \Phi(m)$ is concentrated on the jump set $J_{m}$. For instance the Jin-Kohn entropies (4) are entropies in that sense. Note that here we will be computing entropy productions $\operatorname{div} \Phi(m)$ of unit vector fields $m$ that may not be divergence free, so additional terms involving $\operatorname{div} m$ will appear. But since $\operatorname{div} m \in L_{\text {loc }}^{\frac{4}{3}}$ we can use commutator estimates as described above in the spirit of [DLI15] to deduce that $\operatorname{div} \Phi(m) \in L_{\text {loc }}^{\frac{4}{3}}$ for our map $m$ and any entropy $\Phi$. Refining the commutator estimates from [DLI15, we obtain in fact a more precise pointwise bound. Specifically, for all $C^{2}$ entropies $\Phi$, we have

$$
|\operatorname{div} \Phi(m)| \lesssim\|\Phi\|_{C^{2}\left(\mathbb{S}^{1}\right)} \mathcal{P} \quad \text { a.e., for some } \mathcal{P} \in L_{\text {loc }}^{\frac{4}{3}}
$$

This is achieved in Section 3 ,

Note that to obtain (14) we mollify $m$ and since the mollified $m_{\varepsilon}$ take values into $\bar{B}_{1}$ instead of $\mathbb{S}^{1}$, one first needs to extend $\Phi$ to $\bar{B}_{1}$. For (14) the choice of an extension is quite flexible, but the rest of our proof relies crucially on choosing extensions with specific algebraic properties to obtain more information. A key observation in [LP18] is that a special family of extended entropies $\widetilde{\Phi}: \mathbb{R}^{2} \rightarrow \mathbb{R}^{2}$, called harmonic entropies, satisfy the identity

$$
\begin{aligned}
\operatorname{div} \widetilde{\Phi}(m)=A^{\widetilde{\Phi}}(m) \operatorname{div} m & +F_{1}^{\widetilde{\Phi}}(m) \operatorname{div} \Sigma_{1}(m) \\
& +F_{2}^{\widetilde{\Phi}}(m) \operatorname{div} \Sigma_{2}(m) \quad \text { if } m: \mathbb{R}^{2} \rightarrow \mathbb{S}^{1} \text { is smooth, }
\end{aligned}
$$

where $A^{\widetilde{\Phi}}, F_{1}^{\widetilde{\Phi}}$ and $F_{2}^{\widetilde{\Phi}}$ are smooth functions depending on the harmonic entropy $\widetilde{\Phi}$. Using (15) and commutator estimates and recalling that $\operatorname{div} \Sigma_{j}(m)=0$, we are then able to compute

$$
\operatorname{div} \widetilde{\Phi}(m)=A^{\widetilde{\Phi}}(m) \operatorname{div} m \quad \text { a.e., }
$$


for our map $m$. We prove this identity in Section 4

The conclusion of the proof follows in Section 5, where we remark that for any fixed $x$, the explicit linear map $\widetilde{\Phi} \mapsto A^{\widetilde{\Phi}}(m(x))$ can not satisfy a bound of the form $\left|A^{\widetilde{\Phi}}(m(x))\right| \lesssim\left\|\widetilde{\Phi}_{\left[\mathbb{S}^{1}\right.}\right\|_{C^{2}\left(\mathbb{S}^{1}\right)}$. This is related to the fact that the Hilbert transform (or conjugate function operator) on $\mathbb{S}^{1}$ is not bounded from $C^{0}\left(\mathbb{S}^{1}\right)$ to $L^{\infty}\left(\mathbb{S}^{1}\right)$ (see e.g. Zyg02, $\left.\S \mathrm{VII}\right]$ ). As a consequence, the only possibility for (16) and (14) to be compatible is that div $m=0$ a.e., and we are then in a situation to apply the rigidity result in [LP18.

Acknowledgements. We warmly thank the anonymous referee for suggesting significant simplifications, in particular pointing out that one of our key arguments could be directly formulated in terms of the Hilbert transform without resorting to superfluous intermediate steps. A.L. gratefully acknowledges the support of the Simons foundation, collaboration grant \#426900. X.L. was supported in part by ANR project ANR-18-CE40-0023 and COOPINTER project IEA-297303.

\section{Control of $\operatorname{div} m$ in $L^{\frac{4}{3}}$}

In this section we obtain some preliminary $L^{\frac{4}{3}}$ control of $\operatorname{div} m$ for $m$ satisfying the assumptions of Theorem 1. Let $\rho$ be the standard convolution kernel, and let $\rho_{\varepsilon}(z)=\rho\left(\frac{z}{\varepsilon}\right) \varepsilon^{-2}$. Given a function $f$ we let $[f]_{\varepsilon}:=f * \rho_{\varepsilon}$. Let us first recall the definition of Besov spaces on domains Tri06]. Given a bounded Lipschitz domain $\Omega \subset \mathbb{R}^{n}$ and $f: \Omega \rightarrow \mathbb{R}, z \in \mathbb{R}^{n}$, we define

$$
D^{z} f(x):= \begin{cases}f(x+z)-f(x) & \text { if } x, x+z \in \Omega \\ 0 & \text { otherwise. }\end{cases}
$$

For any $s \in(0,1) p, q \in[1, \infty]$, we set

$$
|f|_{B_{p, q}^{s}(\Omega)}=\left\|t^{-s} \sup _{|h| \leq t}\right\| D^{h} f\left\|_{L^{p}(\Omega)}\right\|_{L^{q}\left(\frac{d t}{t}\right)},
$$

and the Besov space $B_{p, q}^{s}(\Omega)$ is the space of functions $f: \Omega \rightarrow \mathbb{R}$ such that

$$
\|f\|_{L^{p}(\Omega)}+|f|_{B_{p, q}^{s}(\Omega)}<\infty .
$$

In the sequel we will mostly use the space $B_{4, \infty}^{\frac{1}{3}}$. The main result of this section is

Proposition 6. Let $m: \Omega \rightarrow \mathbb{R}^{2}$ satisfy (5), then

$$
m \in B_{4, \infty, \text { loc }}^{\frac{1}{3}}(\Omega) \quad \text { and } \quad \operatorname{div} m \in L_{\mathrm{loc}}^{\frac{4}{3}}(\Omega) .
$$

Moreover we have

$$
|\operatorname{div} m| \lesssim \mathcal{P} \quad \text { a.e. in } \Omega \text {, }
$$

where $\mathcal{P} \in L_{\mathrm{loc}}^{\frac{4}{3}}(\Omega)$ is any weak $L_{\mathrm{loc}}^{\frac{4}{3}}$ limit of a subsequence of

$$
\mathcal{P}_{\varepsilon}(x):=\varepsilon^{-1} f_{B_{\varepsilon}(0)}\left|D^{z} m(x)\right|^{3} d z,
$$

as $\varepsilon \rightarrow 0$.

Let us briefly sketch the proof of Proposition 6. A key role is played by the identity

$$
\begin{aligned}
\operatorname{div} w= & -2 w_{1} w_{2} \operatorname{div} \Sigma_{1}(w) \\
& +\left(w_{1}^{2}-w_{2}^{2}\right) \operatorname{div} \Sigma_{2}(w) \\
& +L(w)[\nabla w]\left(1-|w|^{2}\right) \quad \text { for } w: \mathbb{R}^{2} \rightarrow \mathbb{R}^{2} \text { smooth }
\end{aligned}
$$


where $L(w)$ is a linear form on $\mathbb{R}^{2 \times 2}$ which depends smoothly on $w$. After applying this identity to the mollified map $w=m_{\varepsilon}$, we show that the right-hand side is a sum of terms which vanish as $\varepsilon \rightarrow 0$ in $\mathcal{D}^{\prime}(\Omega)$, and terms which are bounded pointwise by $\mathcal{P}_{\varepsilon}$. Upon proving that $\mathcal{P}_{\varepsilon}$ is bounded in $L_{\text {loc }}^{\frac{4}{3}}$, we are then able to conclude. These facts are a consequence of a preliminary regularity estimate: our

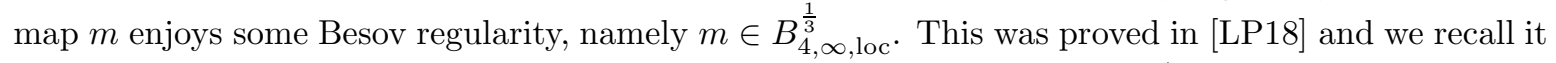
in Lemma 7 . This Besov regularity directly implies that $\mathcal{P}_{\varepsilon}$ is bounded in $L_{\text {loc }}^{\frac{4}{3}}$, as shown in Lemma 8 , Then the bounds on all above mentioned terms rely on pointwise commutator estimates. Specifically, we prove in Lemma 9 that

$$
\left|[\Pi(m)]_{\varepsilon}-\Pi\left(m_{\varepsilon}\right)\right|\left|\nabla m_{\varepsilon}\right| \lesssim \mathcal{P}_{\varepsilon}(x) \quad \text { for smooth maps } \Pi .
$$

Choosing $\Pi=|\cdot|^{2}$, this obviously enables us to estimate $L\left(m_{\varepsilon}\right)\left[\nabla m_{\varepsilon}\right]\left(1-\left|m_{\varepsilon}\right|^{2}\right)$. Moreover, remarking that the condition $\operatorname{div} \Sigma_{j}(m)=0$ ensures that

$$
\operatorname{div} \Sigma_{j}\left(m_{\varepsilon}\right)=\operatorname{div}\left[\left(\Sigma_{j}\left(m_{\varepsilon}\right)\right)-\left[\Sigma_{j}(m)\right]_{\varepsilon}\right],
$$

we are also able to deal with the other terms, which are of the form

$$
\begin{aligned}
F_{j}\left(m_{\varepsilon}\right) \operatorname{div} \Sigma_{j}\left(m_{\varepsilon}\right)= & \operatorname{div}\left[\left(F_{j}\left(m_{\varepsilon}\right)\left(\left(\Sigma_{j}\left(m_{\varepsilon}\right)\right)-\left[\Sigma_{j}(m)\right]_{\varepsilon}\right)\right]\right. \\
& -D F_{j}\left(m_{\varepsilon}\right)\left[\nabla m_{\varepsilon}\right]\left(\left(\Sigma_{j}\left(m_{\varepsilon}\right)\right)-\left[\Sigma_{j}(m)\right]_{\varepsilon}\right),
\end{aligned}
$$

for some smooth $F_{j}: \mathbb{R}^{2} \rightarrow \mathbb{R}$. The first term is easily seen to go to zero in $\mathcal{D}^{\prime}(\Omega)$, while the second term is bounded by $\mathcal{P}_{\varepsilon}$ thanks to the pointwise commutator estimate with $\Pi=\Sigma_{j}$.

Before turning to the full proof of Proposition 6 we gather the intermediate results Lemmas 7,8 and 9 below. In Lemma 7 we recall from [LP18] the regularity $m \in B_{4, \infty, \text { loc }}^{\frac{1}{3}}$ In Lemma 8 we infer from this that $\mathcal{P}_{\varepsilon}$ is bounded in $L_{\text {loc }}^{\frac{4}{3}}$. And in Lemma 9 we establish the above mentioned pointwise commutator estimates.

Lemma 7 ([LP18, Theorem 4]). Let $m: \Omega \rightarrow \mathbb{R}^{2}$ satisfy (5), then $m \in B_{4, \infty, \text { loc }}^{\frac{1}{3}}(\Omega)$.

Proof of Lemma 7. This is essentially proved in [LP18, Theorem 4]. For the reader's convenience we reproduce the argument here, adopting, for the sake of variety, a slightly different point of view.

Since div $\Sigma_{j}(m)=0$, we infer that $\operatorname{curl}\left(i \Sigma_{j}(m)\right)=0$. Recall that from Remark 3 we are assuming $\Omega$ is simply connected, and thus there exists $F_{j}: \Omega \rightarrow \mathbb{R}$ with

$$
\nabla F_{j}=i \Sigma_{j}(m) \quad \text { a.e. }
$$

Since $|m|=1$ a.e. we may choose $\theta: \Omega \rightarrow \mathbb{R}$ such that $m=e^{i \theta}$ a.e., and by definition (6)-(7) of $P$ and $K$ it follows that $F=\left(F_{1}, F_{2}\right)$ satisfies

$$
D F=\left(\begin{array}{c}
i \Sigma_{1}(m) \\
i \Sigma_{2}(m)
\end{array}\right)=P(\theta) \in K \quad \text { a.e. }
$$

For any given $U \subset \subset \Omega$ and $h \in \mathbb{R}^{2}$ with $|h|$ sufficiently small, e.g. $|h|<\frac{1}{3} \operatorname{dist}(U, \partial \Omega)$, by [LP18, Lemma 7] we have

$$
\operatorname{det}(D F(x+h)-D F(x)) \geq c_{0}|D F(x+h)-D F(x)|^{4}
$$

for some constant $c_{0}>0$ and a.e. $x \in \Omega$ with $\operatorname{dist}(x, \partial \Omega)>|h|$. By definition of $F$ in (19) we have

$$
\operatorname{det}(D F(\cdot+h)-D F)=\left(i D^{h} \Sigma_{1}(m)\right) \cdot\left(D^{h} \Sigma_{2}(m)\right) \stackrel{(19)}{=} D^{h} \nabla F_{1} \cdot D^{h} \Sigma_{2}(m),
$$

and also

$$
\left|D^{h} m(x)\right| \lesssim|D F(x+h)-D F(x)| .
$$


Hence gathering the three above equations, we obtain

$$
\left|D^{h} m\right|^{4} \lesssim D^{h} \nabla F_{1} \cdot D^{h} \Sigma_{2}(m) \quad \text { for a.e. } x \in \Omega \text { with } \operatorname{dist}(x, \partial \Omega)>|h| .
$$

Let $\eta \in C_{c}^{\infty}(\Omega)$ be a test function with $\operatorname{dist}(\operatorname{supp} \eta, \partial \Omega)>2 h$ and $\mathbb{1}_{U} \leq \eta \leq \mathbb{1}_{\Omega}$. Integrating by parts and using that $\operatorname{div} \Sigma_{2}(m)=0$ (and thus $\int_{\Omega} \nabla\left(D^{h} F_{1} \eta^{2}\right) \cdot D^{h} \Sigma_{2}(m) d x=0$ ), we have

$$
\begin{aligned}
\int_{\Omega} \eta^{2} D^{h} \nabla F_{1} \cdot D^{h} \Sigma_{2}(m) d x & =-\int_{\Omega} D^{h} F_{1} D^{h} \Sigma_{2}(m) \cdot \nabla\left(\eta^{2}\right) d x \\
& \lesssim|h|\left\|\nabla F_{1}\right\|_{L^{\infty}(\Omega)}\left\|\nabla \Sigma_{2}\right\|_{L^{\infty}\left(\bar{B}_{1}\right)}\|\nabla \eta\|_{L^{\infty}(\Omega)} \int_{\Omega}|\eta|\left|D^{h} m\right| d x \\
& \lesssim|h|\|\nabla \eta\|_{L^{\infty}(\Omega)}\left(\int_{\Omega} \eta^{2}\left|D^{h} m\right|^{4} d x\right)^{\frac{1}{4}} .
\end{aligned}
$$

Recalling (20) we deduce

$$
\int_{\Omega} \eta^{2}\left|D^{h} m\right|^{4} d x \lesssim|h|\|\nabla \eta\|_{L^{\infty}(\Omega)}\left(\int_{\Omega} \eta^{2}\left|D^{h} m\right|^{4} d x\right)^{\frac{1}{4}}
$$

and thus

$$
\left(\int_{\Omega} \eta^{2}\left|D^{h} m\right|^{4} d x\right)^{\frac{1}{4}} \lesssim|h|^{\frac{1}{3}}\|\nabla \eta\|_{L^{\infty}(\Omega)}^{\frac{1}{3}} .
$$

As $\mathbb{1}_{U} \leq \eta$, it follows that

$$
t^{-\frac{1}{3}} \sup _{|h| \leq t}\left\|D^{h} m\right\|_{L^{4}(U)} \lesssim\|\nabla \eta\|_{L^{\infty}(\Omega)}^{\frac{1}{3}}
$$

for $t$ sufficiently small. For larger $t$ values, the boundedness of $m$ implies that $t^{-\frac{1}{3}} \sup _{|h| \leq t}\left\|D^{h} m\right\|_{L^{4}(U)}$ is bounded. Thus $m \in B_{4, \infty}^{\frac{1}{3}}(U)$ for all $U \subset \subset \Omega$.

Lemma 8. Given $m \in B_{4, \infty, \text { loc }}^{\frac{1}{3}}(\Omega)$, let $\mathcal{P}_{\varepsilon}$ be as in (18). Then for any $U \subset \subset \Omega$ and $\varepsilon$ sufficiently small we have

$$
\left\|\mathcal{P}_{\varepsilon}\right\|_{L^{\frac{4}{3}}(U)} \leq|m|_{B_{4, \infty}^{\frac{1}{3}}(U)}^{3} .
$$

In particular $\mathcal{P}_{\varepsilon}$ is bounded in $L_{\mathrm{loc}}^{\frac{4}{3}}(\Omega)$.

Proof of Lemma 8, Jensen's inequality implies

$$
\begin{aligned}
\int_{U}\left(\mathcal{P}_{\varepsilon}\right)^{\frac{4}{3}} d x & \stackrel{\sqrt{(18)}}{=} \varepsilon^{-\frac{4}{3}} \int_{U}\left(f_{B_{\varepsilon}(0)}\left|D^{z} m(x)\right|^{3} d z\right)^{\frac{4}{3}} d x \\
& \leq \varepsilon^{-\frac{4}{3}} \int_{U} f_{B_{\varepsilon}(0)}\left|D^{z} m(x)\right|^{4} d z d x \\
& \leq \varepsilon^{-\frac{4}{3}} \sup _{|z| \leq \varepsilon} \int_{U}\left|D^{z} m(x)\right|^{4} d x,
\end{aligned}
$$

which gives (21). 
Lemma 9. Let $m: \Omega \rightarrow \mathbb{R}^{2}$ be such that $|m| \leq R$ a.e. for some $0<R<\infty$ and $\Pi \in C^{2}\left(\mathbb{R}^{2} ; \mathbb{R}\right)$. For any $x \in \Omega$ such that $\bar{B}_{\varepsilon}(x) \subset \Omega$, we have

$$
\left|[\Pi(m)]_{\varepsilon}(x)-\Pi\left(m_{\varepsilon}(x)\right)\right|\left|\nabla m_{\varepsilon}(x)\right| \lesssim\left\|\nabla^{2} \Pi\right\|_{L^{\infty}\left(\bar{B}_{R}\right)} \mathcal{P}_{\varepsilon}(x),
$$

where $\mathcal{P}_{\varepsilon}$ is as in (18).

Proof of Lemma 9, Let $x \in \Omega$ be such that $\bar{B}_{\varepsilon}(x) \subset \Omega$. The commutator estimate (54) proved in Appendix A. Lemma 17 gives

$$
\left|[\Pi(m)]_{\varepsilon}(x)-\Pi\left(m_{\varepsilon}(x)\right)\right| \lesssim\left\|D^{2} \Pi\right\|_{L^{\infty}\left(\bar{B}_{R}\right)} f_{B_{\varepsilon}(0)}\left|D^{z} m(x)\right|^{2} d z,
$$

so Jensen's inequality and the definition (18) of $\mathcal{P}_{\varepsilon}$ imply

$$
\begin{aligned}
\left|[\Pi(m)]_{\varepsilon}(x)-\Pi\left(m_{\varepsilon}(x)\right)\right|^{\frac{3}{2}} & \lesssim\left\|D^{2} \Pi\right\|_{L^{\infty}\left(\bar{B}_{R}\right)}^{\frac{3}{2}} f_{B_{\varepsilon}(0)}\left|D^{z} m(x)\right|^{3} d z \\
& \stackrel{18}{=}\left\|D^{2} \Pi\right\|_{L^{\infty}\left(\bar{B}_{R}\right)}^{\frac{3}{2}} \varepsilon \mathcal{P}_{\varepsilon}(x) .
\end{aligned}
$$

To estimate $\nabla m_{\varepsilon}$ we compute, using the fact that $\nabla \rho_{\varepsilon}$ has zero average,

$$
\nabla m_{\varepsilon}(x)=\int_{B_{\varepsilon}(0)} m(x-z) \nabla \rho_{\varepsilon}(z) d z=\int_{B_{\varepsilon}(0)} D^{-z} m(x) \nabla \rho_{\varepsilon}(z) d z
$$

Hence by Jensen's inequality and (18) again,

$$
\left|\nabla m_{\varepsilon}(x)\right|^{3} \lesssim \varepsilon^{-3} f_{B_{\varepsilon}(0)}\left|D^{-z} m(x)\right|^{3} d z=\varepsilon^{-2} \mathcal{P}_{\varepsilon}(x) .
$$

From (23)-(24) we gather

$$
\begin{aligned}
\left|[\Pi(m)]_{\varepsilon}(x)-\Pi\left(m_{\varepsilon}(x)\right)\right|\left|\nabla m_{\varepsilon}(x)\right| & \lesssim\left(\left\|D^{2} \Pi\right\|_{L^{\infty}\left(\bar{B}_{R}\right)}^{\frac{3}{2}} \varepsilon \mathcal{P}_{\varepsilon}(x)\right)^{\frac{2}{3}}\left(\varepsilon^{-2} \mathcal{P}_{\varepsilon}(x)\right)^{\frac{1}{3}} \\
& \lesssim\left\|D^{2} \Pi\right\|_{L^{\infty}\left(\bar{B}_{R}\right)} \mathcal{P}_{\varepsilon}(x)
\end{aligned}
$$

Equipped with Lemmas 7,8 and 9 we can now prove Proposition 6 .

Proof of Proposition [6. First note that by Lemmas 7 and 8 we have $m \in B_{4, \infty, \text { loc }}^{\frac{1}{3}}(\Omega)$ and $\mathcal{P}_{\varepsilon}$ is bounded in $L_{\text {loc }}^{\frac{4}{3}}(\Omega)$, hence it admits weakly converging subsequences.

Then the strategy of the proof involves the convoluted map $m_{\varepsilon}$, for which we can use the chain rule to compute $\operatorname{div} \Sigma_{k}\left(m_{\varepsilon}\right)$. Using some algebraic identities specific to the Jin-Kohn entropies and the commutator estimates of Lemma 9 this enables us to control div $m_{\varepsilon}$ in terms of $\mathcal{P}_{\varepsilon}$. 
For any smooth function $w: \Omega \rightarrow \mathbb{R}^{2}$,

$$
\begin{aligned}
& \operatorname{div} \Sigma_{1}(w) \stackrel{(4)}{=} \partial_{1} w_{2}\left(1-w_{1}^{2}-\frac{w_{2}^{2}}{3}\right)+w_{2}\left(-2 w_{1} \partial_{1} w_{1}-\frac{2}{3} w_{2} \partial_{1} w_{2}\right) \\
& +\partial_{2} w_{1}\left(1-w_{2}^{2}-\frac{w_{1}^{2}}{3}\right)+w_{1}\left(-2 w_{2} \partial_{2} w_{2}-\frac{2}{3} w_{1} \partial_{2} w_{1}\right) \\
& =-2 w_{1} w_{2} \operatorname{div} w+\left(\partial_{1} w_{2}+\partial_{2} w_{1}\right)\left(1-|w|^{2}\right), \\
& \operatorname{div} \Sigma_{2}(w) \stackrel{\text { 函 }}{=}-\partial_{1} w_{1}\left(1-\frac{2}{3} w_{1}^{2}\right)-w_{1}\left(-\frac{4}{3} w_{1} \partial_{1} w_{1}\right) \\
& +\partial_{2} w_{2}\left(1-\frac{2}{3} w_{2}^{2}\right)+w_{2}\left(-\frac{4}{3} w_{2} \partial_{2} w_{2}\right) \\
& =-\partial_{1} w_{1}\left(1-2 w_{1}^{2}\right)+\partial_{2} w_{2}\left(1-2 w_{2}^{2}\right) \\
& =-\partial_{1} w_{1}\left(1-2 w_{1}^{2}\right)+\left(\operatorname{div} w-\partial_{1} w_{1}\right)\left(1-2 w_{2}^{2}\right) \\
& =-\partial_{1} w_{1}\left(2-2 w_{1}^{2}-2 w_{2}^{2}\right)+\operatorname{div} w\left(1-2 w_{2}^{2}\right) \\
& =\left(w_{1}^{2}-w_{2}^{2}\right) \operatorname{div} w+\left(\partial_{2} w_{2}-\partial_{1} w_{1}\right)\left(1-|w|^{2}\right) .
\end{aligned}
$$

Multiplying (25) by $-2 w_{1} w_{2}$, (26) by $\left(w_{1}^{2}-w_{2}^{2}\right)$ and adding the resulting identities, we infer

$\operatorname{div} w=G_{1}(w) \operatorname{div} \Sigma_{1}(w)+G_{2}(w) \operatorname{div} \Sigma_{2}(w)+L(w)[\nabla w]\left(1-|w|^{2}\right)$,

where

$$
G_{1}(w)=-2 w_{1} w_{2}, \quad G_{2}(w)=w_{1}^{2}-w_{2}^{2},
$$

and

$$
\begin{aligned}
L(w)[\nabla w]= & \left(1+|w|^{2}\right) \operatorname{div} w+2 w_{1} w_{2}\left(\partial_{1} w_{2}+\partial_{2} w_{1}\right) \\
& +\left(w_{2}^{2}-w_{1}^{2}\right)\left(\partial_{2} w_{2}-\partial_{1} w_{1}\right) .
\end{aligned}
$$

Note that $L(w)$ is a linear form on $\mathbb{R}^{2 \times 2}$ which depends smoothly on $w$, and that $G_{1}, G_{2}$ are smooth functions of $w$. We fix $\Omega^{\prime} \subset \subset \Omega$ and apply this to $w=m_{\varepsilon}$. Thus for small enough $\varepsilon$,

$$
\begin{aligned}
\operatorname{div} m_{\varepsilon}= & G_{1}\left(m_{\varepsilon}\right) \operatorname{div} \Sigma_{1}\left(m_{\varepsilon}\right)+G_{2}\left(m_{\varepsilon}\right) \operatorname{div} \Sigma_{2}\left(m_{\varepsilon}\right) \\
& +L\left(m_{\varepsilon}\right)\left[\nabla m_{\varepsilon}\right]\left(1-\left|m_{\varepsilon}\right|^{2}\right) \quad \text { in } \Omega^{\prime} .
\end{aligned}
$$

Recalling that $\operatorname{div} \Sigma_{k}(m)=0$ for $k \in\{1,2\}$, we also have

$$
\operatorname{div}\left(\left[\Sigma_{k}(m)\right]_{\varepsilon}\right)=\left[\operatorname{div} \Sigma_{k}(m)\right]_{\varepsilon}=0 \quad \text { in } \Omega^{\prime},
$$

and may therefore (using also that $|m|=1$ a.e.) rewrite (27) as

$$
\operatorname{div} m_{\varepsilon}=A_{1}^{\varepsilon}+A_{2}^{\varepsilon}+B^{\varepsilon}+R_{1}^{\varepsilon}+R_{2}^{\varepsilon},
$$

where

$$
\begin{aligned}
& A_{k}^{\varepsilon}=-D G_{k}\left(m_{\varepsilon}\right)\left[\nabla m_{\varepsilon}\right] \cdot\left(\Sigma_{k}\left(m_{\varepsilon}\right)-\left[\Sigma_{k}(m)\right]_{\varepsilon}\right) \quad \text { for } k=1,2, \\
& B^{\varepsilon}=L\left(m_{\varepsilon}\right)\left[\nabla m_{\varepsilon}\right]\left(1-\left|m_{\varepsilon}\right|^{2}\right)=L\left(m_{\varepsilon}\right)\left[\nabla m_{\varepsilon}\right]\left(\left[|m|^{2}\right]_{\varepsilon}-\left|m_{\varepsilon}\right|^{2}\right), \\
& R_{k}^{\varepsilon}=\operatorname{div}\left[G_{k}\left(m_{\varepsilon}\right)\left(\Sigma_{k}\left(m_{\varepsilon}\right)-\left[\Sigma_{k}(m)\right]_{\varepsilon}\right)\right] \quad \text { for } k=1,2 .
\end{aligned}
$$


For $k \in\{1,2\}$, because $\Sigma_{k}$ is smooth and $m \in L^{\infty}(\Omega)$ we have $\Sigma_{k}\left(m_{\varepsilon}\right) \rightarrow \Sigma_{k}(m)$ and $\left[\Sigma_{k}(m)\right]_{\varepsilon} \rightarrow$ $\Sigma_{k}(m)$ strongly in $L^{p}\left(\Omega^{\prime}\right)$ for any $p \in[1, \infty)$, and in particular

$$
\begin{aligned}
& \Sigma_{k}\left(m_{\varepsilon}\right)-\left[\Sigma_{k}(m)\right]_{\varepsilon} \\
& =\Sigma_{k}\left(m_{\varepsilon}\right)-\Sigma_{k}(m)+\Sigma_{k}(m)-\left[\Sigma_{k}(m)\right]_{\varepsilon} \longrightarrow 0 \quad \text { strongly in } L^{1}\left(\Omega^{\prime}\right) .
\end{aligned}
$$

Since $\left|m_{\varepsilon}\right| \leq 1$ and $G_{k}$ is smooth this implies

$$
R_{k}^{\varepsilon}=\operatorname{div}\left[G_{k}\left(m_{\varepsilon}\right)\left(\Sigma_{k}\left(m_{\varepsilon}\right)-\left[\Sigma_{k}(m)\right]_{\varepsilon}\right)\right] \longrightarrow 0 \quad \text { in } \mathcal{D}^{\prime}\left(\Omega^{\prime}\right) .
$$

Next we notice that $A_{1}^{\varepsilon}+A_{2}^{\varepsilon}+B^{\varepsilon}$ in (28) is a sum of terms of the form

$$
X^{\varepsilon}=T\left(m_{\varepsilon}\right)\left[\nabla m_{\varepsilon}\right]\left([\Pi(m)]_{\varepsilon}-\Pi\left(m_{\varepsilon}\right)\right),
$$

for some smooth functions $\Pi$ and linear forms $T\left(m_{\varepsilon}\right)$ depending smoothly on $m_{\varepsilon}$. Recalling again that $\left|m_{\varepsilon}\right| \leq 1$, Lemma 9 therefore ensures that

$$
\left|A_{1}^{\varepsilon}+A_{2}^{\varepsilon}+B^{\varepsilon}\right| \lesssim \mathcal{P}_{\varepsilon} \quad \text { in } \Omega^{\prime} .
$$

Plugging (30) and (29) into (28) and recalling that $m_{\varepsilon} \rightarrow m$ in $L^{1}\left(\Omega^{\prime}\right)$ we infer

$$
\begin{aligned}
\left|\int_{\Omega} m \cdot \nabla \zeta d x\right| & =\liminf _{\varepsilon \rightarrow 0}\left|\int_{\Omega} m_{\varepsilon} \cdot \nabla \zeta d x\right| \\
& \lesssim \liminf _{\varepsilon \rightarrow 0} \int_{\Omega}|\zeta| \mathcal{P}_{\varepsilon} d x \quad \text { for all } \zeta \in C_{c}^{\infty}\left(\Omega^{\prime}\right)
\end{aligned}
$$

Thanks to Lemma 8 we may choose a sequence $\varepsilon_{n} \rightarrow 0$ such that $\mathcal{P}_{\varepsilon_{n}} \rightarrow \mathcal{P}$ weakly in $L_{\text {loc }}^{\frac{4}{3}}$. Then (31) gives

$$
\left|\int_{\Omega} m \cdot \nabla \zeta d x\right| \lesssim \int_{\Omega} \mathcal{P}|\zeta| d x
$$

In particular

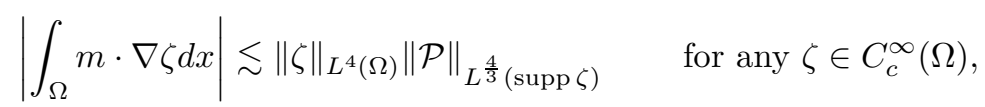

which implies that $\operatorname{div} m \in L_{\text {loc }}^{\frac{4}{3}}(\Omega)$. Taking $\zeta=\mathbb{1}_{U} * \rho_{\delta}$ and letting $\delta \rightarrow 0$ gives

$$
\left|\int_{U} \operatorname{div} m d x\right| \lesssim \int_{\bar{U}} \mathcal{P} d x \quad \text { for all } U \subset \subset \Omega .
$$

Now choosing $U=B_{r}(x)$ and letting $r \rightarrow 0$ we get (17) for all Lebesgue points of $\operatorname{div} m$ and $\mathcal{P}$.

\section{Control of all entropy productions}

Recall that an entropy is a smooth map $\Phi: \mathbb{S}^{1} \rightarrow \mathbb{R}^{2}$ that satisfies $e^{i t} \cdot \frac{d}{d t} \Phi\left(e^{i t}\right)=0$ for all $t \in \mathbb{R}$.

Proposition 10. Let $m: \Omega \rightarrow \mathbb{R}^{2}$ satisfy (5). For any entropy $\Phi \in C^{2}\left(\mathbb{S}^{1}\right)$ we have $\operatorname{div} \Phi(m) \in L_{\text {loc }}^{\frac{4}{3}}(\Omega)$ and

$$
|\operatorname{div} \Phi(m)(x)| \lesssim\|\Phi\|_{C^{2}\left(\mathbb{S}^{1}\right)} \mathcal{P}(x) \quad \text { for a.e. } x \in \Omega,
$$

where $\mathcal{P} \in L_{\mathrm{loc}}^{\frac{4}{3}}(\Omega)$ is as in Proposition 6 . 
The proof of Proposition 10 is a refinement of the argument in [DLI15, Proposition 3], replacing their commutator estimates by the pointwise commutator estimates proved in Lemma 9, Section 2 ,

Proof of Proposition 10, Let $\eta:[0, \infty) \rightarrow \mathbb{R}$ be a cut-off function with $\eta=0$ on $\left[0, \frac{1}{2}\right] \cup[2, \infty), \eta(1)=1$, and define

$$
\widetilde{\Phi}(z):=\eta(|z|) \Phi\left(\frac{z}{|z|}\right) .
$$

By Step 2 of the proof of [DLI15, Proposition 3] we have that

$$
D \widetilde{\Phi}(z)=-2 \Psi(z) \otimes z+\gamma(z) I d \quad \text { for every } z \in \mathbb{R}^{2}
$$

where $I d$ is the identity matrix and

$$
\gamma(z)=\frac{z^{\perp} \cdot D \widetilde{\Phi}(z) z^{\perp}}{|z|^{2}} \text { and } \Psi(z)=\frac{-D \widetilde{\Phi}(z) z+\gamma(z) z}{2|z|^{2}} .
$$

Note that

$$
\|\gamma\|_{W^{1, \infty}\left(\mathbb{R}^{2}\right)} \lesssim\left(\left\|D^{2} \widetilde{\Phi}\right\|_{L^{\infty}\left(\mathbb{R}^{2}\right)}+\|D \widetilde{\Phi}\|_{L^{\infty}\left(\mathbb{R}^{2}\right)}\right) \lesssim\|\Phi\|_{C^{2}\left(\mathbb{S}^{1}\right)}
$$

and hence

$$
\|D \Psi\|_{L^{\infty}\left(\mathbb{R}^{2}\right)} \stackrel{\sqrt[34]{34}, \sqrt{33}}{\lesssim}\|\Phi\|_{C^{2}\left(\mathbb{S}^{1}\right)} .
$$

Exactly as in Step 3 of the proof of [DLI15, Proposition 3] we see that

$$
\operatorname{div} \widetilde{\Phi}\left(m_{\varepsilon}\right)=\Psi\left(m_{\varepsilon}\right) \cdot \nabla\left(1-\left|m_{\varepsilon}\right|^{2}\right)+\gamma\left(m_{\varepsilon}\right) \operatorname{div} m_{\varepsilon} .
$$

Testing with $\zeta \in C_{c}^{\infty}(\Omega)$ we have

$$
\begin{aligned}
& \int_{\Omega} \widetilde{\Phi}\left(m_{\varepsilon}\right) \cdot \nabla \zeta d x \\
& \stackrel{\sqrt[(36)]{=}}{=} \int_{\Omega}\left(1-\left|m_{\varepsilon}\right|^{2}\right) \Psi\left(m_{\varepsilon}\right) \cdot \nabla \zeta d x \\
& \quad+\int_{\Omega}\left(\Psi_{1,1}\left(m_{\varepsilon}\right) m_{\varepsilon 1,1}+\Psi_{1,2}\left(m_{\varepsilon}\right) m_{\varepsilon 2,1}\right. \\
& \left.\quad+\Psi_{2,1}\left(m_{\varepsilon}\right) m_{\varepsilon 1,2}+\Psi_{2,2}\left(m_{\varepsilon}\right) m_{\varepsilon 2,2}\right)\left(1-\left|m_{\varepsilon}\right|^{2}\right) \zeta d x \\
& \quad-\int_{\Omega} \gamma\left(m_{\varepsilon}\right) \operatorname{div} m_{\varepsilon} \zeta d x .
\end{aligned}
$$

Note that by Proposition [6, $\operatorname{div} m_{\varepsilon} \rightarrow \operatorname{div} m$ in $L_{\text {loc }}^{\frac{4}{3}}(\Omega)$. Thus, choosing a sequence $\varepsilon_{n}$ such that $\mathcal{P}_{\varepsilon_{n}} \rightarrow \mathcal{P}$ weakly in $L_{\text {loc }}^{\frac{4}{3}}$, we obtain

$$
\begin{aligned}
& \left|\int_{\Omega} \Phi(m) \cdot \nabla \zeta d x\right|=\lim _{n \rightarrow \infty}\left|\int_{\Omega} \widetilde{\Phi}\left(m_{\varepsilon_{n}}\right) \cdot \nabla \zeta d x\right| \\
& \left.\stackrel{\text { (37), (34), 351) }}{\lesssim}\|\Phi\|_{C^{2}\left(\mathbb{S}^{1}\right)} \lim _{n \rightarrow \infty} \int_{\Omega}\left|\nabla m_{\varepsilon_{n}}\right||1-| m_{\varepsilon_{n}}\right|^{2}|| \zeta \mid d x \\
& +\|\Phi\|_{C^{2}\left(\mathbb{S}^{1}\right)} \lim _{n \rightarrow \infty} \int_{\Omega}\left|\operatorname{div} m_{\varepsilon_{n}}\right||\zeta| d x \\
& \stackrel{[221,(17])}{\lesssim}\|\Phi\|_{C^{2}\left(\mathbb{S}^{1}\right)} \int_{\Omega} \mathcal{P}|\zeta| d x \quad \text { for all } \zeta \in C_{c}^{\infty}(\Omega) \text {. }
\end{aligned}
$$


Since $\mathcal{P} \in L_{\text {loc }}^{\frac{4}{3}}(\Omega)$ this implies that $\operatorname{div} \Phi(m) \in L_{\text {loc }}^{\frac{4}{3}}(\Omega)$. Further (32) follows from the above and the same arguments presented at the end of the proof of Proposition 6 .

Remark 11. Later on we are going to specialize to entropies $\Phi$ of the form

$$
\Phi\left(e^{i t}\right)=\psi(t) e^{i t}+\psi^{\prime}(t) i e^{i t},
$$

for some $\psi \in C^{3}\left(\mathbb{S}^{1}\right) \approx C^{3}(\mathbb{R} / 2 \pi \mathbb{Z})$. Then the control established in Proposition 10 becomes

$$
|\operatorname{div} \Phi(m)(x)| \lesssim \mathcal{P}(x)\|\psi\|_{C^{3}\left(\mathbb{S}^{1}\right)} \quad \text { for a.e. } x \in \Omega .
$$

\section{Computation of harmonic entropy productions}

In [DMKO01], entropies were first defined as smooth maps $\Phi: \mathbb{R}^{2} \rightarrow \mathbb{R}^{2}$ that satisfy $e^{i t} \cdot \frac{d}{d t} \Phi\left(r e^{i t}\right)=0$ for all $t \in \mathbb{R}$ and $r>0$. Such entropies can be obtained from smooth functions $\varphi$ via the formula

$$
\Phi^{\varphi}(z)=\varphi(z) z+((i z) \cdot \nabla \varphi(z)) i z \quad \forall z \in \mathbb{R}^{2} .
$$

In [LP18, the second two authors introduced the notion of harmonic entropies, which are entropies $\Phi$ given by harmonic functions $\varphi$ through (39). They enjoy nice factorization properties with respect to the Jin-Kohn entropies, and this fact was a major ingredient in LP18.

While the entropy production $\operatorname{div} \Phi(m)$ only depends on the values of $\Phi$ on $\mathbb{S}^{1}$, for the purpose of estimating $\operatorname{div} \Phi\left(m_{\varepsilon}\right)$ one needs $\Phi$ to be extended outside $\mathbb{S}^{1}$ (as in the proof of Proposition [10). Since $\left|m_{\varepsilon}\right| \leq 1$ it is however enough to specify values of $\Phi \in \bar{B}_{1}$ (rather than all of $\mathbb{R}^{2}$ as the entropies used in [DMKO01, LP18]).

In this section, we use the nice factorization properties of harmonic entropies given by (39) for $\varphi \in C^{3}\left(\bar{B}_{1}\right)$ harmonic in $B_{1}$, and obtain an explicit pointwise expression for the associated entropy productions.

For any function $\psi \in L^{2}\left(\mathbb{S}^{1}\right)$ we denote by $E \psi$ its harmonic extension, that is, $E: L^{2}\left(\mathbb{S}^{1}\right) \rightarrow L^{2}\left(B_{1}\right)$ is the continuous linear operator uniquely determined by its action on Fourier modes

$$
E \psi_{k}\left(r e^{i \theta}\right)=r^{|k|} e^{i k \theta}, \quad \text { for } \psi_{k}(\theta)=e^{i k \theta} .
$$

In what follows we will use only the basic fact that $E$ is continuous from $C^{4}\left(\mathbb{S}^{1}\right)$ to $C^{3}\left(\bar{B}_{1}\right)$ (see the proof of Lemma 16).

Proposition 12. Let $m$ satisfy (5). Given any $\psi \in C^{4}\left(\mathbb{S}^{1}\right)$ and $\varphi=E \psi$ its harmonic extension, the harmonic entropy given by

$$
\Phi^{E \psi}(z)=\Phi^{\varphi}(z)=\varphi(z) z+((i z) \cdot \nabla \varphi(z)) i z \quad \forall z \in \bar{B}_{1},
$$

satisfies

$$
\operatorname{div} \Phi^{E \psi}(m)(x)=\mathcal{A} \psi(m(x)) \operatorname{div} m(x) \quad \text { for a.e. } x \in \Omega,
$$

where $\mathcal{A}: C^{4}\left(\mathbb{S}^{1}\right) \rightarrow C^{0}\left(\mathbb{S}^{1}\right)$ is the Fourier multiplier operator given by

$$
\mathcal{A} \psi_{k}=\frac{|k|^{3}-2 k^{2}-|k|+2}{2} \psi_{k}, \quad \psi_{k}(\theta)=e^{i k \theta} \text { for all } k \in \mathbb{Z} .
$$

Remark 13. The Fourier multiplier operator $\mathcal{A}$ is naturally defined on complex-valued functions but in (41) only its restriction to real-valued functions plays a role. 
Remark 14. The Fourier multiplier operator $\mathcal{A}$ defined by (42) is well-defined and continuous from $C^{4}\left(\mathbb{S}^{1}\right)$ to $C^{0}\left(\mathbb{S}^{1}\right)$. To show this, note that the Fourier coefficients of $\psi=\sum_{k} c_{k}(\psi) \psi_{k} \in C^{4}\left(\mathbb{S}^{1}\right)$ satisfy $k^{4}\left|c_{k}(\psi)\right|=\left|c_{k}\left(\psi^{(4)}\right)\right| \in \ell^{2}$. Noting the fact that $\left|c_{k}(\psi)\right|\left|\mathcal{A}\left(\psi_{k}\right)\right| \lesssim \frac{1}{k}\left|c_{k}(\psi)\right| k^{4}$ this implies, via Cauchy-Schwarz' inequality in $\ell^{2}$, that the Fourier series defining $\mathcal{A} \psi$ converges uniformly (so $\mathcal{A}$ is well-defined) and gives the continuity estimate $\|\mathcal{A} \psi\|_{C^{0}\left(\mathbb{S}^{1}\right)} \lesssim\left|c_{0}(\psi)\right|+\left\|\psi^{(4)}\right\|_{L^{2}\left(\mathbb{S}^{1}\right)} \lesssim\|\psi\|_{C^{4}\left(\mathbb{S}^{1}\right)}$. For finer boundedness properties of Fourier multiplier operators see e.g. Gra08.

We split the proof of Proposition 12 into Lemmas [15] and 16. The most crucial one is Lemma [15, where we rely on arguments from [LP18] to explicitly compute $\operatorname{div} \Phi(m)$ for any harmonic entropy $\Phi$. Then in Lemma 16 we use this computation and the definition of the harmonic extension operator to obtain (41).

Lemma 15. Let $m$ satisfy (5). Let $\varphi \in C^{3}\left(\bar{B}_{1}\right)$ be such that $\Delta \varphi=0$ in $B_{1}$ and $\Phi^{\varphi}$ be the corresponding harmonic entropy given by

$$
\Phi^{\varphi}=\varphi(z) z+((i z) \cdot \nabla \varphi(z)) i z \quad \forall z \in \bar{B}_{1} .
$$

Then we have

$$
\operatorname{div} \Phi^{\varphi}(m)=A^{\varphi}(m) \operatorname{div} m \quad \text { a.e. in } \Omega,
$$

where $A^{\varphi} \in C^{0}\left(\bar{B}_{1}\right)$ is given by

$$
\begin{aligned}
A^{\varphi}(z)= & \varphi(z)-z_{1} \partial_{1} \varphi(z)-z_{2} \partial_{2} \varphi(z) \\
& +z_{1} z_{2}\left[\partial_{12} \varphi(z)-z_{2} \partial_{111} \varphi(z)+z_{1} \partial_{211} \varphi(z)\right] \\
& +\frac{1}{2}\left(z_{1}^{2}-z_{2}^{2}\right)\left[\partial_{11} \varphi(z)+z_{2} \partial_{112} \varphi(z)+z_{1} \partial_{111} \varphi(z)\right] .
\end{aligned}
$$

Proof of Lemma 15. The convoluted map $m_{\varepsilon}$ is smooth with values into $\bar{B}_{1}$, and a direct computation (to be found in Appendix B. Lemma 18) shows that

$$
\operatorname{div} \Phi^{\varphi}\left(m_{\varepsilon}\right)=A^{\varphi}\left(m_{\varepsilon}\right) \operatorname{div} m_{\varepsilon}+R_{\varepsilon}^{0}+R_{\varepsilon}^{1}+R_{\varepsilon}^{2},
$$

where

$$
\begin{aligned}
& R_{\varepsilon}^{0}=\operatorname{div}\left(\left(\left|m_{\varepsilon}\right|^{2}-1\right) B^{\varphi}\left(m_{\varepsilon}\right)\right), \\
& R_{\varepsilon}^{j}=F_{j}^{\varphi}\left(m_{\varepsilon}\right) \operatorname{div} \Sigma_{j}\left(m_{\varepsilon}\right) \quad \text { for } j=1,2,
\end{aligned}
$$

where $B^{\varphi}: \bar{B}_{1} \rightarrow \mathbb{R}^{2}$ and $F_{1}^{\varphi}, F_{2}^{\varphi}: \bar{B}_{1} \rightarrow \mathbb{R}$ are continuous and depend only on $\varphi$.

Since $m \in L^{\infty}$ we have $m_{\varepsilon} \rightarrow m$ in $L^{p}(\Omega)$ for all $p \in[1, \infty)$. In particular, $\Phi^{\varphi}$ being $C^{2}$, this implies that $\Phi^{\varphi}\left(m_{\varepsilon}\right) \rightarrow \Phi^{\varphi}(m)$ in $L^{1}(\Omega)$ and therefore

$$
\operatorname{div} \Phi^{\varphi}\left(m_{\varepsilon}\right) \longrightarrow \operatorname{div} \Phi^{\varphi}(m) \quad \text { in } \mathcal{D}^{\prime}(\Omega) .
$$

Recall that $\operatorname{div} m \in L_{\text {loc }}^{\frac{4}{3}}$ by Proposition [6] thus $\operatorname{div} m_{\varepsilon} \rightarrow \operatorname{div} m$ in $L_{\text {loc }}^{\frac{4}{3}}$. Since $A^{\varphi}: \bar{B}_{1} \rightarrow \mathbb{R}$ is continuous, $m_{\varepsilon} \rightarrow m$ a.e. and $\left|m_{\varepsilon}\right| \leq 1$, by dominated convergence we have $A^{\varphi}\left(m_{\varepsilon}\right) \rightarrow A^{\varphi}(m)$ in $L^{4}$ and we deduce

$$
A^{\varphi}\left(m_{\varepsilon}\right) \operatorname{div} m_{\varepsilon} \longrightarrow A^{\varphi}(m) \operatorname{div} m \quad \text { in } L_{\mathrm{loc}}^{1}(\Omega) \text { and hence in } \mathcal{D}^{\prime}(\Omega) .
$$

Similarly we have $\left(\left|m_{\varepsilon}\right|^{2}-1\right) B^{\varphi}\left(m_{\varepsilon}\right) \rightarrow 0$ in $L^{1}(\Omega)$, and

$$
R_{\varepsilon}^{0} \longrightarrow 0 \quad \text { in } \mathcal{D}^{\prime}(\Omega)
$$


Hence to conclude the proof of Lemma 15 it suffices to show that

$$
R_{\varepsilon}^{j} \longrightarrow 0 \quad \text { in } \mathcal{D}^{\prime}(\Omega) \text { for } j=1,2,
$$

to pass to the limit in (44) and to use $\operatorname{div} m \in L_{\text {loc }}^{\frac{4}{3}}(\Omega)$.

The proof of (45) follows the ideas of [LP18, Section 6], with slight modifications. It relies on two crucial ingredients: the vanishing of the Jin-Kohn entropy productions $\operatorname{div} \Sigma_{j}(m)=0$ for $j=1,2$; and the regularity $m \in B_{4, \infty, \text { loc }}^{\frac{1}{3}}$, as used also in Section 2 ,

Let $j \in\{1,2\}$. Using the explicit expression of $\operatorname{div} \Sigma_{j}\left(m_{\varepsilon}\right)$ obtained from (25)-(26), we have

$$
\left|\operatorname{div} \Sigma_{j}\left(m_{\varepsilon}\right)\right| \lesssim\left|\operatorname{div} m_{\varepsilon}\right|+\left|\nabla m_{\varepsilon}\right|\left(1-\left|m_{\varepsilon}\right|^{2}\right) .
$$

Recall from Proposition 6 that $\operatorname{div} m \in L_{\mathrm{loc}}^{\frac{4}{3}}$, and from Lemma 9 (applied to $\Pi=|\cdot|^{2}$ ) that

$$
\left|\nabla m_{\varepsilon}\right|\left(1-\left|m_{\varepsilon}\right|^{2}\right) \lesssim \mathcal{P}_{\varepsilon}
$$

Since $\mathcal{P}_{\varepsilon}$ is bounded in $L_{\text {loc }}^{\frac{4}{3}}$ (see Lemma 8 ), we deduce from the above that $\operatorname{div} \Sigma_{j}\left(m_{\varepsilon}\right)$ is bounded in $L_{\text {loc }}^{\frac{4}{3}}$. Because we also have $\operatorname{div} \Sigma_{j}\left(m_{\varepsilon}\right) \rightarrow \operatorname{div} \Sigma_{j}(m)=0$ in $\mathcal{D}^{\prime}(\Omega)$, we infer

$$
\operatorname{div} \Sigma_{j}\left(m_{\varepsilon}\right) \rightarrow 0 \quad \text { in } L_{\mathrm{loc}}^{\frac{4}{3}}(\Omega) .
$$

Combined with the fact that $F_{j}^{\varphi}\left(m_{\varepsilon}\right)$ converges strongly to $F_{j}^{\varphi}(m)$ in $L_{\text {loc }}^{4}(\Omega)$, this implies (invoking e.g. [Bre11, Proposition 3.5(iv)])

$$
F_{j}^{\varphi}\left(m_{\varepsilon}\right) \operatorname{div} \Sigma_{j}\left(m_{\varepsilon}\right) \rightarrow 0 \quad \text { in } L_{\mathrm{loc}}^{1}(\Omega),
$$

which proves (45).

The second step towards Proposition 12 is to obtain an expression of $A^{\varphi}$ in terms of the Fourier coefficients of $\varphi_{\mathbb{S}^{1}}$. While Lemma 15] naturally defines $A^{\varphi}$ for real-valued functions $\varphi$ (which give rise to $\mathbb{R}^{2}$-valued entropies $\Phi^{\varphi}$ ), formula (43) also makes sense for complex-valued functions and in what follows it is convenient to consider $A^{\varphi}$ to be extended to complex-valued functions $\varphi$ through (43).

Lemma 16. The operator

$$
\mathcal{A}: C^{4}\left(\mathbb{S}^{1}\right) \longrightarrow C^{0}\left(\mathbb{S}^{1}\right), \quad \psi \longmapsto A_{\left[\mathbb{S}^{1}\right.}^{E \psi}
$$

is the Fourier multiplier operator characterized by

$$
\mathcal{A} \psi_{k}=\frac{|k|^{3}-2 k^{2}-|k|+2}{2} \psi_{k}, \quad \text { for all } k \in \mathbb{Z},
$$

where $\psi_{k}(\theta)=e^{i k \theta}$.

Proof of Lemma [16. The linear operator $\mathcal{A}$ defined via (46) is continuous from $C^{4}\left(\mathbb{S}^{1}\right)$ to $C^{0}\left(\mathbb{S}^{1}\right)$ thanks to the bounds

$$
\|\mathcal{A} \psi\|_{C^{0}\left(\mathbb{S}^{1}\right)} \lesssim\|E \psi\|_{C^{3}\left(\bar{B}_{1}\right)} \lesssim\|\psi\|_{C^{4}\left(\mathbb{S}^{1}\right)} .
$$

The first inequality follows directly from the explicit definition of $A^{\varphi}$ (43). The second is a consequence of $\left\|E \psi_{k}\right\|_{C^{3}\left(\bar{B}_{1}\right)} \lesssim 1+|k|^{3}$ and of the estimates in Remark 14. Moreover, as shown in Remark 14, the Fourier multiplier operator $\mathcal{B}$ defined by the right-hand side of (47) is continuous from $C^{4}\left(\mathbb{S}^{1}\right)$ to $C^{0}\left(\mathbb{S}^{1}\right)$. The two linear continuous operators $\mathcal{A}$ and $\mathcal{B}$ agree on $C^{4}\left(\mathbb{S}^{1}\right)$ provided they agree on the Fourier modes $\psi_{k}$, whose linear span is dense. Hence we only need to show (47) for each fixed $k \in \mathbb{Z}$. 
For $k \geq 1$ the harmonic extension of $\psi_{k}$ is $E \psi_{k}=\varphi_{k}$ (40) where $\varphi_{k}(z)=z^{k}$ (here we identify $\mathbb{R}^{2}$ and $\mathbb{C}$ ), so $\partial_{1} \varphi_{k}=k \varphi_{k-1}$ and $\partial_{2} \varphi_{k}=i k \varphi_{k-1}$. For $k \geq 3$ we may thus compute

$$
\begin{aligned}
A^{\varphi_{k}}= & \varphi_{k}-k\left(z_{1} \varphi_{k-1}+i z_{2} \varphi_{k-1}\right) \\
& +z_{1} z_{2}\left(i k(k-1) \varphi_{k-2}-z_{2} k(k-1)(k-2) \varphi_{k-3}+i z_{1} k(k-1)(k-2) \varphi_{k-3}\right) \\
& +\frac{1}{2}\left(z_{1}^{2}-z_{2}^{2}\right)\left(k(k-1) \varphi_{k-2}+i z_{2} k(k-1)(k-2) \varphi_{k-3}+z_{1} k(k-1)(k-2) \varphi_{k-3}\right) \\
= & (1-k) \varphi_{k}+i z_{1} z_{2} k(k-1)^{2} \varphi_{k-2}+\frac{1}{2}\left(z_{1}^{2}-z_{2}^{2}\right) k(k-1)^{2} \varphi_{k-2} .
\end{aligned}
$$

To obtain the last equality we used that

$$
\left(z_{1}+i z_{2}\right) \varphi_{j-1}=z \varphi_{j-1}=\varphi_{j} \quad \text { for } j=k \text { and } k-2 .
$$

Computing further and using that $z^{2} \varphi_{k-2}=\varphi_{k}$ we find

$$
\begin{aligned}
A^{\varphi_{k}} & =(1-k) \varphi_{k}+\frac{1}{2} k(k-1)^{2}\left(z_{1}+i z_{2}\right)^{2} \varphi_{k-2} \\
& =\left(1-k+\frac{1}{2} k(k-1)^{2}\right) \varphi_{k}=\frac{k^{3}-2 k^{2}-k+2}{2} \varphi_{k} .
\end{aligned}
$$

Since $\varphi_{k}=E \psi_{k}$ this proves (47) for $k \geq 3$. Similar computations show that (47) is valid for $0 \leq k \leq 2$. For $k<0$ we obtain (47) by remarking that $\varphi_{k}$ is the complex conjugate of $\varphi_{-k}$ and therefore $A^{\varphi_{k}}$ is the complex conjugate of $A^{\varphi-k}$.

Proposition 12 follows directly from Lemmas 15 and 16.

\section{Proof of Theorems 1 and 2}

The proof of Theorem 1 reduces to showing $\operatorname{div} m=0$, which then allows us to invoke the main theorem in [LP18] to conclude the rigidity of $m$. To this end we compare, on the one hand the pointwise control

$$
\left|\operatorname{div} \Phi^{E \psi}(m)(x)\right| \lesssim\|\psi\|_{C^{3}} \mathcal{P}(x)
$$

obtained in Proposition 10 (see also Remark 11), and on the other hand the explicit expression

$$
\operatorname{div} \Phi^{E \psi}(m)(x)=\mathcal{A} \psi(m(x)) \operatorname{div} m(x),
$$

obtained in Proposition 12 for some explicit Fourier multiplier operator $\mathcal{A}$. We show indeed that this operator can not satisfy a bound of the form $\left|\mathcal{A} \psi\left(z_{0}\right)\right| \lesssim\|\psi\|_{C^{3}\left(\mathbb{S}^{1}\right)}$ for any $z_{0} \in \mathbb{S}^{1}$, because this would imply that the Hilbert transform $\mathcal{H}$ on the circle (or conjugate function transform), defined on Fourier modes $\psi_{k}=e^{i k \theta}$ by $\mathcal{H} \psi_{k}=-i \operatorname{sign}(k) \psi_{k}$, is bounded from $C^{0}\left(\mathbb{S}^{1}\right)$ into $L^{\infty}\left(\mathbb{S}^{1}\right)$, and this is known to be wrong. Therefore, the only way for the pointwise control of $\operatorname{div} \Phi^{E \psi}(m)$ in terms of $\|\psi\|_{C^{3}}$ to be valid is that $\operatorname{div} m=0$. In addition to this basic argument, some technicalities enter the game due to the fact that the pointwise control and explicit expression of Propositions 10 and 12 are "almost everywhere" statements and the set of points $x$ at which they are valid depends in principle on $\psi$. We classically circumvent this by arguing on countable families of $\psi$ with appropriate density properties.

Proof of Theorem [1. Let $\mathcal{X} \subset C^{4}\left(\mathbb{S}^{1}\right)$ denote a countable dense subset. Let $\mathcal{G} \subset \Omega$ be the set of all points $x \in \Omega$ at which $\mathcal{P}(x)<\infty$, and both:

- the explicit expression

$$
\operatorname{div} \Phi^{E \psi}(m)(x)=\mathcal{A} \psi(m(x)) \operatorname{div} m(x),
$$

given by (41), 
- its pointwise control

$$
\left|\operatorname{div} \Phi^{E \psi}(m)(x)\right| \lesssim \mathcal{P}(x)\|\psi\|_{C^{3}\left(\mathbb{S}^{1}\right)},
$$

provided by (38),

are satisfied for all $\psi \in \mathcal{X}$. Thanks to Proposition 12 and Proposition 10, the set $\mathcal{G}$ is a countable intersection of sets of full measure, and therefore $|\Omega \backslash \mathcal{G}|=0$.

We claim that $\operatorname{div} m(x)=0$ for all $x \in \mathcal{G}$. Assume by contradiction that $\operatorname{div} m\left(x_{0}\right) \neq 0$ for some $x_{0} \in \mathcal{G}$. By definition of $\mathcal{G}$ we have

$$
\left|\mathcal{A} \psi\left(m\left(x_{0}\right)\right) \operatorname{div} m\left(x_{0}\right)\right|=\left|\operatorname{div} \Phi^{E \psi}(m)\left(x_{0}\right)\right| \leq C\|\psi\|_{C^{3}\left(\mathbb{S}^{1}\right)},
$$

for all $\psi \in \mathcal{X}$ and some constant $C=C\left(x_{0}, m\right)>0$.

In what follows we identify $\mathbb{S}^{1}$ with $\mathbb{R} / 2 \pi \mathbb{Z}$, functions on $\mathbb{S}^{1}$ with $2 \pi$-periodic functions on $\mathbb{R}$, and $m\left(x_{0}\right) \in \mathbb{S}^{1}$ with its argument $\theta_{0} \in \mathbb{R} / 2 \pi \mathbb{Z}$. Dividing (48) by $\operatorname{div} m\left(x_{0}\right) \neq 0$ we deduce

$$
\left|\mathcal{A} \psi\left(\theta_{0}\right)\right| \leq \widetilde{C}\|\psi\|_{C^{3}\left(\mathbb{S}^{1}\right)}
$$

for some other constant $\widetilde{C}=\widetilde{C}\left(x_{0}, m\right)>0$ and all $\psi \in \mathcal{X}$. Since $\mathcal{X}$ is dense in $C^{4}\left(\mathbb{S}^{1}\right)$ and both sides of the above estimate depend continuously on $\psi$ in the $C^{4}$ topology, we infer that

$$
\left|\mathcal{A} \psi\left(\theta_{0}\right)\right| \leq \widetilde{C}\|\psi\|_{C^{3}\left(\mathbb{S}^{1}\right)} \quad \forall \psi \in C^{4}\left(\mathbb{S}^{1}\right) .
$$

The multiplier operator $\mathcal{A}$ commutes with translations of the variable and this automatically turns estimate (49) at a fixed $\theta_{0}$ into an estimate at any $\theta \in \mathbb{R}$. Explicitly, for any $\psi \in C^{4}\left(\mathbb{S}^{1}\right)$ and $\varpi \in \mathbb{R}$ we may apply (49) to the translated function $\psi^{\varpi}=\psi(\cdot+\varpi)$. Since $\mathcal{A} \psi^{\varpi}=\mathcal{A} \psi(\cdot+\varpi)$ (as can be checked on Fourier modes, see e.g. [Gra08, § 3.6.1]), we obtain $\left|\mathcal{A} \psi\left(\theta_{0}+\varpi\right)\right| \leq \widetilde{C}\|\psi\|_{C^{3}\left(\mathbb{S}^{1}\right)}$ for any $\psi$ and $\varpi$ and deduce

$$
\|\mathcal{A} \psi\|_{L^{\infty}\left(\mathbb{S}^{1}\right)} \leq \widetilde{C}\|\psi\|_{C^{3}\left(\mathbb{S}^{1}\right)} \quad \forall \psi \in C^{4}\left(\mathbb{S}^{1}\right) .
$$

Splitting $\mathcal{A}=\frac{1}{2} \mathcal{A}_{0}+\frac{1}{2} \mathcal{A}_{1}$ where

$$
\mathcal{A}_{0} \psi_{k}=|k|^{3} \psi_{k}, \quad \text { and } \quad \mathcal{A}_{1} \psi_{k}=\left(2-2 k^{2}-|k|\right) \psi_{k},
$$

we see (arguing as in Remark 14) that $\mathcal{A}_{1}$ defines a continuous linear operator from $C^{3}\left(\mathbb{S}^{1}\right)$ to $C^{0}\left(\mathbb{S}^{1}\right)$, and therefore (50) implies that

$$
\left\|\mathcal{A}_{0} \psi\right\|_{L^{\infty}\left(\mathbb{S}^{1}\right)} \lesssim\|\psi\|_{C^{3}\left(\mathbb{S}^{1}\right)} \quad \forall \psi \in C^{4}\left(\mathbb{S}^{1}\right) .
$$

Remarking that $\mathcal{A}_{0} \psi=-\mathcal{H} \psi^{(3)}$, where $\mathcal{H}$ is the Hilbert transform on the circle (or conjugate function transform, see [Gra08, $\S 3.5]$ ), that is, the Fourier multiplier operator given by

$$
\mathcal{H} \psi_{0}=0, \quad \mathcal{H} \psi_{k}=-i \operatorname{sign}(k) \psi_{k} \quad \forall k \in \mathbb{Z} \backslash\{0\},
$$

we obtain the estimate

$$
\left\|\mathcal{H} \psi^{(3)}\right\|_{L^{\infty}\left(\mathbb{S}^{1}\right)} \stackrel{\sqrt[511]{\lesssim}}{\lesssim}\|\psi\|_{C^{3}\left(\mathbb{S}^{1}\right)} \quad \forall \psi \in C^{4}\left(\mathbb{S}^{1}\right),
$$

which readily turns into

$$
\|\mathcal{H} \psi\|_{L^{\infty}\left(\mathbb{S}^{1}\right)} \lesssim\|\psi\|_{C^{0}\left(\mathbb{S}^{1}\right)} \quad \forall \psi \in C^{1}\left(\mathbb{S}^{1}\right) .
$$

Since $\mathcal{H}: C^{0}\left(\mathbb{S}^{1}\right) \rightarrow L^{2}\left(\mathbb{S}^{1}\right)$ is continuous and $C^{1}\left(\mathbb{S}^{1}\right)$ is dense in $C^{0}\left(\mathbb{S}^{1}\right)$, this estimate implies that $\mathcal{H}$ maps in fact $C^{0}\left(\mathbb{S}^{1}\right)$ continuously into $L^{\infty}\left(\mathbb{S}^{1}\right)$, which is notoriously wrong (see e.g. Zyg02, $\S$ VII.2], the function $\psi(\theta)=\sum_{k \geq 2} \sin (k \theta) /(k \log k)$ is continuous but its Hilbert transform $\mathcal{H} \psi(\theta)=$ $-\sum_{k \geq 2} \cos (k \theta) /(k \log k)$ is not bounded $)$.

This concludes the proof that $\operatorname{div} m=0$ a.e. Now $m$ indeed satisfies the assumptions of [LP18, Theorem 3], which gives the desired rigidity for $m$. 
The proof of Theorem 2 relies on the correspondence between solutions of the differential inclusion $D F \in K$ a.e. and unit vector fields $m$ satisfying the assumptions of Theorem 1

Proof of Theorem 圆. Let $F: \Omega \rightarrow \mathbb{R}^{2}$ be a Lipschitz map such that $D F \in K$ a.e., and we define $m: \Omega \rightarrow \mathbb{R}^{2}$ by

$$
m_{1}=-F_{1,1}-F_{2,2}, \quad m_{2}=F_{1,2}-F_{2,1} .
$$

For $x \in \Omega$ such that $D F(x)=P(\theta) \in K$, where recall that $P: \mathbb{R} \rightarrow K \subset \mathbb{R}^{2 \times 2}$ is the parameterization of the set $K$ defined in (6), it is clear from (52) and (6) that $m=e^{i \theta}$. Further

$$
i \Sigma_{j}(m(x))=\nabla F_{j}(x) \quad \text { for } j=1,2,
$$

where $\Sigma_{1}, \Sigma_{2}$ are the Jin-Kohn entropies defined in (4). Thus the vector field $m$ defined by (52) satisfies

$$
|m(x)|=1 \text { a.e. } \quad \text { and } \operatorname{div}\left(\Sigma_{j}(m)\right) \stackrel{(53)}{=} \operatorname{curl}\left(\nabla F_{j}\right)=0 \text { for } j=1,2 .
$$

By Theorem 1, $m$ is locally Lipschitz outside a locally finite set of points $S$. From (53) we deduce that $D F$ agrees almost everywhere with a map $G$ that is locally Lipschitz outside of $S$, and therefore $D F$ itself is locally Lipschitz outside of $S$ (indeed outside of $S$ the map $G$ is locally the gradient of a function with Lipschitz derivatives, which has to agree with $F$ up to a constant). Moreover in any convex neighborhood of a point in $S, m$ is a vortex, which translates into (8).

Finally, Theorem 5 is a reformulation of Theorem 2 by identifying $v=F_{1}+i F_{2}$ as in the proof of Theorem 5 in LP18. Hence $v$ and $F$ have the same regularity.

\section{A A Commutator estimate}

In this appendix we prove the following basic commutator estimate:

Lemma 17. Given $\Pi \in C^{2}\left(\mathbb{R}^{2}\right)$ and $m: \Omega \rightarrow \mathbb{R}^{2}$ with $|m| \leq R$ a.e. for some $0<R<\infty$, we have

$$
\left|[\Pi(m)]_{\varepsilon}(x)-\Pi\left(m_{\varepsilon}(x)\right)\right| \lesssim\left\|D^{2} \Pi\right\|_{L^{\infty}\left(\bar{B}_{R}\right)} f_{B_{\varepsilon}(0)}\left|D^{z} m(x)\right|^{2} d z
$$

Proof of Lemma 17. The proof follows computations presented in [CET94] and recently in a context closer to ours also in DLI15. We write out

$$
\begin{aligned}
& {[\Pi(m)]_{\varepsilon}(x)-\Pi\left(m_{\varepsilon}(x)\right)} \\
& =\int\left(\Pi(m(x-z))-\Pi\left(m_{\varepsilon}(x)\right)\right) \rho_{\varepsilon}(z) d z \\
& =\int D \Pi(m(x-z)) \cdot\left(m(x-z)-m_{\varepsilon}(x)\right) \rho_{\varepsilon}(z) d z \\
& +\int\left(\Pi(m(x-z))-\Pi\left(m_{\varepsilon}(x)\right)-D \Pi(m(x-z)) \cdot\left(m(x-z)-m_{\varepsilon}(x)\right)\right) \rho_{\varepsilon}(z) d z \\
& =\int\left(D \Pi(m(x-z))-D \Pi\left(m_{\varepsilon}(x)\right)\right) \cdot\left(m(x-z)-m_{\varepsilon}(x)\right) \rho_{\varepsilon}(z) d z \\
& +\int\left(\Pi(m(x-z))-\Pi\left(m_{\varepsilon}(x)\right)-D \Pi(m(x-z)) \cdot\left(m(x-z)-m_{\varepsilon}(x)\right)\right) \rho_{\varepsilon}(z) d z .
\end{aligned}
$$

By Taylor expansion we have

$$
\begin{aligned}
& \left|\Pi(m(x-z))-\Pi\left(m_{\varepsilon}(x)\right)-D \Pi(m(x-z)) \cdot\left(m(x-z)-m_{\varepsilon}(x)\right)\right| \\
& \quad \lesssim\left\|D^{2} \Pi\right\|_{L^{\infty}}\left|m(x-z)-m_{\varepsilon}(x)\right|^{2}, \\
& \quad \lesssim\left\|D^{2} \Pi\right\|_{L^{\infty}}\left|m(x-z)-m_{\varepsilon}(x)\right|^{2},
\end{aligned}
$$


and plugging this into (55),

$$
\left|[\Pi(m)]_{\varepsilon}(x)-\Pi\left(m_{\varepsilon}(x)\right)\right| \lesssim\left\|D^{2} \Pi\right\|_{L^{\infty}} \int_{B_{\varepsilon}(0)}\left|m(x-z)-m_{\varepsilon}(x)\right|^{2} \rho_{\varepsilon}(z) d z .
$$

Moreover by Jensen's inequality we have

$$
\begin{aligned}
& \int_{B_{\varepsilon}(0)}\left|m(x-z)-m_{\varepsilon}(x)\right|^{2} \rho_{\varepsilon}(z) d z \\
& =\int_{B_{\varepsilon}(0)}\left|\int_{B_{\varepsilon}(0)}(m(x-z)-m(x-y)) \rho_{\varepsilon}(y) d y\right|^{2} \rho_{\varepsilon}(z) d z \\
& \lesssim \int_{B_{\varepsilon}(0)} \int_{B_{\varepsilon}(0)}|m(x-z)-m(x-y)|^{2} \rho_{\varepsilon}(y) \rho_{\varepsilon}(z) d y d z \\
& =\int_{B_{\varepsilon}(0)} \int_{B_{\varepsilon}(0)}\left|D^{-z} m(x)-D^{-y} m(x)\right|^{2} \rho_{\varepsilon}(y) \rho_{\varepsilon}(z) d y d z \\
& \lesssim f_{\mathcal{B}_{\varepsilon}(0)}\left|D^{-z} m(x)\right|^{2} d z+f_{\mathcal{B}_{\varepsilon}(0)}\left|D^{-y} m(x)\right|^{2} d y \\
& \lesssim f_{\mathcal{B}_{\varepsilon}(0)}\left|D^{z} m(x)\right|^{2} d z .
\end{aligned}
$$

Plugging this estimate into (56) gives (54).

\section{B Computations needed in the proof of Lemma 15}

Lemma 18. Let $\varphi \in C^{3}\left(\bar{B}_{1}\right)$ such that $\Delta \varphi=0$ in $B_{1}$ and $\Phi^{\varphi}$ the corresponding harmonic entropy given by

$$
\Phi^{\varphi}(z)=\varphi(z) z+((i z) \cdot \nabla \varphi(z)) i z \quad \forall z \in \bar{B}_{1} .
$$

For any smooth map $w: \Omega \rightarrow \bar{B}_{1}$ we have

$$
\begin{aligned}
\operatorname{div} \Phi^{\varphi}(w)= & A(w) \operatorname{div} w+\operatorname{div}\left(\left(|w|^{2}-1\right) B(w)\right) \\
& +\partial_{2} B_{1}(w) \operatorname{div} \Sigma_{1}(w)-\partial_{1} B_{1}(w) \operatorname{div} \Sigma_{2}(w)
\end{aligned}
$$

where $A=A^{\varphi}: \bar{B}_{1} \rightarrow \mathbb{R}$ and $B=B^{\varphi}: \bar{B}_{1} \rightarrow \mathbb{R}^{2}$ are given by

$$
\begin{aligned}
A^{\varphi}(z)= & \varphi(z)-z_{1} \partial_{1} \varphi(z)-z_{2} \partial_{2} \varphi(z) \\
& +z_{1} z_{2}\left[\partial_{12} \varphi(z)-z_{2} \partial_{111} \varphi(z)+z_{1} \partial_{211} \varphi(z)\right] \\
& +\frac{1}{2}\left(z_{1}^{2}-z_{2}^{2}\right)\left[\partial_{11} \varphi(z)+z_{2} \partial_{112} \varphi(z)+z_{1} \partial_{111} \varphi(z)\right], \\
B^{\varphi}(z)= & \left(\begin{array}{c}
\partial_{1} \varphi(z)+\frac{1}{2} z_{2} \partial_{12} \varphi(z)-\frac{1}{2} z_{1} \partial_{22} \varphi(z) \\
\partial_{2} \varphi(z)-\frac{1}{2} z_{2} \partial_{11} \varphi(z)+\frac{1}{2} z_{1} \partial_{12} \varphi(z)
\end{array}\right) .
\end{aligned}
$$

Proof of Lemma 18, We have

$$
\Phi^{\varphi}(w) \stackrel{(57\rceil)}{=}\left(\begin{array}{c}
w_{1} \varphi-w_{2}\left(-w_{2} \partial_{1} \varphi+w_{1} \partial_{2} \varphi\right) \\
w_{2} \varphi+w_{1}\left(-w_{2} \partial_{1} \varphi+w_{1} \partial_{2} \varphi\right)
\end{array}\right) .
$$


So

$$
\begin{aligned}
\operatorname{div} & \Phi^{\varphi}(w) \\
= & w_{1} \partial_{1} \varphi \partial_{1} w_{1}+w_{1} \partial_{2} \varphi \partial_{1} w_{2}+\varphi \partial_{1} w_{1} \\
& -\left(-w_{2} \partial_{1} \varphi+w_{1} \partial_{2} \varphi\right) \partial_{1} w_{2} \\
& -w_{2}\left(-\partial_{1} \varphi \partial_{1} w_{2}-w_{2} \partial_{11} \varphi \partial_{1} w_{1}-w_{2} \partial_{12} \varphi \partial_{1} w_{2}\right. \\
& \left.\quad+\partial_{2} \varphi \partial_{1} w_{1}+w_{1} \partial_{12} \varphi \partial_{1} w_{1}+w_{1} \partial_{22} \varphi \partial_{1} w_{2}\right) \\
& +w_{2} \partial_{1} \varphi \partial_{2} w_{1}+w_{2} \partial_{2} \varphi \partial_{2} w_{2}+\varphi \partial_{2} w_{2} \\
& +\left(-w_{2} \partial_{1} \varphi+w_{1} \partial_{2} \varphi\right) \partial_{2} w_{1} \\
& +w_{1}\left(-\partial_{1} \varphi \partial_{2} w_{2}-w_{2} \partial_{11} \varphi \partial_{2} w_{1}-w_{2} \partial_{12} \varphi \partial_{2} w_{2}\right. \\
& \left.\quad+\partial_{2} \varphi \partial_{2} w_{1}+w_{1} \partial_{12} \varphi \partial_{2} w_{1}+w_{1} \partial_{22} \varphi \partial_{2} w_{2}\right) \\
= & \left(\varphi+w_{1} \partial_{1} \varphi-w_{2} \partial_{2} \varphi+w_{2}^{2} \partial_{11} \varphi-w_{1} w_{2} \partial_{12} \varphi\right) \partial_{1} w_{1} \\
& +\left(\varphi+w_{2} \partial_{2} \varphi-w_{1} \partial_{1} \varphi+w_{1}^{2} \partial_{22} \varphi-w_{1} w_{2} \partial_{12} \varphi\right) \partial_{2} w_{2} \\
& +\left(w_{1} \partial_{2} \varphi+w_{2} \partial_{1} \varphi-w_{1} \partial_{2} \varphi+w_{2}^{2} \partial_{12} \varphi-w_{1} w_{2} \partial_{22} \varphi+w_{2} \partial_{1} \varphi\right) \partial_{1} w_{2} \\
& +\left(w_{2} \partial_{1} \varphi-w_{2} \partial_{1} \varphi+w_{1} \partial_{2} \varphi-w_{1} w_{2} \partial_{11} \varphi+w_{1}^{2} \partial_{12} \varphi+w_{1} \partial_{2} \varphi\right) \partial_{2} w_{1} \\
= & \left(\varphi+w_{1} \partial_{1} \varphi-w_{2} \partial_{2} \varphi+w_{2}^{2} \partial_{11} \varphi-w_{1} w_{2} \partial_{12} \varphi\right) \partial_{1} w_{1} \\
& +\left(\varphi+w_{2} \partial_{2} \varphi-w_{1} \partial_{1} \varphi+w_{1}^{2} \partial_{22} \varphi-w_{1} w_{2} \partial_{12} \varphi\right) \partial_{2} w_{2} \\
& +\left(2 \partial_{1} \varphi+w_{2} \partial_{12} \varphi-w_{1} \partial_{22} \varphi\right) w_{2} \partial_{1} w_{2} \\
& +\left(2 \partial_{2} \varphi-w_{2} \partial_{11} \varphi+w_{1} \partial_{12} \varphi\right) w_{1} \partial_{2} w_{1}
\end{aligned}
$$

Noting that

$$
\partial_{1}\left(\frac{|w|^{2}}{2}\right)-w_{1} \partial_{1} w_{1}=w_{2} \partial_{1} w_{2} \quad \text { and } \quad \partial_{2}\left(\frac{|w|^{2}}{2}\right)-w_{2} \partial_{2} w_{2}=w_{1} \partial_{2} w_{1},
$$

and plugging this into (60),

$$
\begin{aligned}
\operatorname{div} \Phi^{\varphi}(w) & \left(\varphi+w_{1} \partial_{1} \varphi-w_{2} \partial_{2} \varphi+w_{2}^{2} \partial_{11} \varphi-w_{1} w_{2} \partial_{12} \varphi\right. \\
= & \left.\quad-2 w_{1} \partial_{1} \varphi-w_{1} w_{2} \partial_{12} \varphi+w_{1}^{2} \partial_{22} \varphi\right) \partial_{1} w_{1} \\
& +\left(\varphi+w_{2} \partial_{2} \varphi-w_{1} \partial_{1} \varphi+w_{1}^{2} \partial_{22} \varphi-w_{1} w_{2} \partial_{12} \varphi\right. \\
& \left.\quad-2 w_{2} \partial_{2} \varphi-w_{1} w_{2} \partial_{12} \varphi+w_{2}^{2} \partial_{11} \varphi\right) \partial_{2} w_{2} \\
& +\left(\partial_{1} \varphi+\frac{1}{2} w_{2} \partial_{12} \varphi-\frac{1}{2} w_{1} \partial_{22} \varphi\right) \partial_{1}\left(|w|^{2}\right) \\
& +\left(\partial_{2} \varphi-\frac{1}{2} w_{2} \partial_{11} \varphi+\frac{1}{2} w_{1} \partial_{12} \varphi\right) \partial_{2}\left(|w|^{2}\right) \\
= & \left(\varphi-w_{1} \partial_{1} \varphi-w_{2} \partial_{2} \varphi+w_{1}^{2} \partial_{22} \varphi+w_{2}^{2} \partial_{11} \varphi-2 w_{1} w_{2} \partial_{12} \varphi\right) \operatorname{div} w \\
& +\left(\partial_{1} \varphi+\frac{1}{2} w_{2} \partial_{12} \varphi-\frac{1}{2} w_{1} \partial_{22} \varphi\right) \partial_{1}\left(|w|^{2}\right) \\
& +\left(\partial_{2} \varphi-\frac{1}{2} w_{2} \partial_{11} \varphi+\frac{1}{2} w_{1} \partial_{12} \varphi\right) \partial_{2}\left(|w|^{2}\right) \\
= & C(w) \operatorname{div} w+B(w) \cdot \nabla\left(|w|^{2}\right)
\end{aligned}
$$

where $B=B^{\varphi}$ is as in (59) and

$$
C(z)=\varphi-z_{1} \partial_{1} \varphi-z_{2} \partial_{2} \varphi+z_{1}^{2} \partial_{22} \varphi+z_{2}^{2} \partial_{11} \varphi-2 z_{1} z_{2} \partial_{12} \varphi .
$$


We rewrite (61) as

$$
\begin{aligned}
\operatorname{div} \Phi^{\varphi}(w)= & C(w) \operatorname{div} w+\operatorname{div}\left(\left(|w|^{2}-1\right) B(w)\right) \\
& +\left(\partial_{1} B_{1} \partial_{1} w_{1}+\partial_{2} B_{1} \partial_{1} w_{2}+\partial_{1} B_{2} \partial_{2} w_{1}+\partial_{2} B_{2} \partial_{2} w_{2}\right)\left(1-|w|^{2}\right) .
\end{aligned}
$$

Note that

$$
\begin{aligned}
& \partial_{1} B_{1}=\partial_{11} \varphi+\frac{1}{2} z_{2} \partial_{112} \varphi-\frac{1}{2} \partial_{22} \varphi-\frac{1}{2} z_{1} \partial_{221} \varphi, \\
& \partial_{2} B_{2}=\partial_{22} \varphi-\frac{1}{2} z_{2} \partial_{112} \varphi-\frac{1}{2} \partial_{11} \varphi+\frac{1}{2} z_{1} \partial_{221} \varphi, \\
& \text { so } \quad \partial_{1} B_{1}+\partial_{2} B_{2}=\frac{1}{2} \Delta \varphi,
\end{aligned}
$$

and

$$
\begin{aligned}
& \partial_{2} B_{1}=\frac{3}{2} \partial_{12} \varphi+\frac{1}{2} z_{2} \partial_{122} \varphi-\frac{1}{2} z_{1} \partial_{222} \varphi \\
& \partial_{1} B_{2}=\frac{3}{2} \partial_{12} \varphi-\frac{1}{2} z_{2} \partial_{111} \varphi+\frac{1}{2} z_{1} \partial_{112} \varphi \\
& \text { so } \quad \partial_{2} B_{1}-\partial_{1} B_{2}=\frac{1}{2} z_{2}\left(\partial_{1} \Delta \varphi\right)-\frac{1}{2} z_{1}\left(\partial_{2} \Delta \varphi\right) .
\end{aligned}
$$

Since $\varphi$ is harmonic we have from (65) and (67) that

$$
\partial_{1} B_{1}+\partial_{2} B_{2}=0 \quad \text { and } \quad \partial_{2} B_{1}-\partial_{1} B_{2}=0 .
$$

Recalling moreover the explicit expressions of $\operatorname{div} \Sigma_{j}(w)$ computed in (25)-(26), we find that (63) can be rewritten as

$$
\begin{aligned}
& \operatorname{div} \Phi^{\varphi}(w) \stackrel{68],[63]}{=} C(w) \operatorname{div} w+\operatorname{div}\left(\left(|w|^{2}-1\right) B(w)\right) \\
& +\partial_{1} B_{1}\left(\partial_{1} w_{1}-\partial_{2} w_{2}\right)\left(1-|w|^{2}\right) \\
& +\partial_{2} B_{1}\left(\partial_{1} w_{2}+\partial_{2} w_{1}\right)\left(1-|w|^{2}\right) \\
& \stackrel{(25)+(26)}{=} C(w) \operatorname{div} w+\operatorname{div}\left(\left(|w|^{2}-1\right) B(w)\right) \\
& -\partial_{1} B_{1}\left(\operatorname{div} \Sigma_{2}(w)-\left(w_{1}^{2}-w_{2}^{2}\right) \operatorname{div} w\right) \\
& +\partial_{2} B_{1}\left(\operatorname{div} \Sigma_{1}(w)+2 w_{1} w_{2} \operatorname{div} w\right) \\
& =A(w) \operatorname{div} w+\operatorname{div}\left(\left(|w|^{2}-1\right) B\right) \\
& +\partial_{2} B_{1} \operatorname{div} \Sigma_{1}(w)-\partial_{1} B_{1} \operatorname{div} \Sigma_{2}(w),
\end{aligned}
$$


where

$$
\begin{aligned}
A(w)= & C(w)+2 \partial_{2} B_{1} w_{1} w_{2}+\partial_{1} B_{1}\left(w_{1}^{2}-w_{2}^{2}\right) \\
& \stackrel{\text { [66] }}{=}, w_{1} \partial_{1} \varphi-w_{2} \partial_{2} \varphi+w_{1}^{2} \partial_{22} \varphi+w_{2}^{2} \partial_{11} \varphi-2 w_{1} w_{2} \partial_{12} \varphi \\
& +2 w_{1} w_{2}\left(\frac{3}{2} \partial_{12} \varphi+\frac{1}{2} w_{2} \partial_{122} \varphi-\frac{1}{2} w_{1} \partial_{222} \varphi\right) \\
& +\left(w_{1}^{2}-w_{2}^{2}\right)\left(\partial_{11} \varphi+\frac{1}{2} w_{2} \partial_{112} \varphi-\frac{1}{2} \partial_{22} \varphi-\frac{1}{2} w_{1} \partial_{221} \varphi\right) \\
= & \varphi-w_{1} \partial_{1} \varphi-w_{2} \partial_{2} \varphi \\
& +2 w_{1} w_{2}\left(\frac{1}{2} \partial_{12} \varphi+\frac{1}{2} w_{2} \partial_{122} \varphi-\frac{1}{2} w_{1} \partial_{222} \varphi\right) \\
& +\left(w_{1}^{2}-w_{2}^{2}\right)\left(\frac{1}{2} \partial_{11} \varphi+\frac{1}{2} w_{2} \partial_{112} \varphi-\frac{1}{2} w_{1} \partial_{221} \varphi\right) .
\end{aligned}
$$

This expression agrees with (58) because $\Delta \varphi=0$, so (69) proves Lemma 18 ,

\section{References}

$\left[\mathrm{ACF}^{+} 19\right]$ K. Astala, A. Clop, D. Faraco, J. Jääskeläinen, and A. Koski. Improved Hölder regularity for strongly elliptic PDEs. arXiv:1906.10906, 2019.

[ADLM99] L. Ambrosio, C. De Lellis, and C. Mantegazza. Line energies for gradient vector fields in the plane. Calc. Var. Partial Differential Equations, 9(4):327-255, 1999.

[AG87] P. Aviles and Y. Giga. A mathematical problem related to the physical theory of liquid crystal configurations. In Miniconference on geometry and partial differential equations, 2 (Canberra, 1986), volume 12 of Proc. Centre Math. Anal. Austral. Nat. Univ., pages 1-16. Austral. Nat. Univ., Canberra, 1987.

[AG96] P. Aviles and Y. Giga. The distance function and defect energy. Proc. Roy. Soc. Edinburgh Sect. A, 126(5):923-938, 1996.

[AIM09] K. Astala, T. Iwaniec, and G. Martin. Elliptic Partial Differential Equations and Quasiconformal Mappings in the Plane, volume 48 of Princeton Mathematical Series. Princeton University Press, 2009.

[AIS01] K. Astala, T. Iwaniec, and E. Saksman. Beltrami operators in the plane. Duke Math.J., $107,2001$.

[AKLR02] L. Ambrosio, B. Kirchheim, M. Lecumberry, and T. Rivière. On the rectifiability of defect measures arising in a micromagnetics model. In Nonlinear problems in mathematical physics and related topics, II, volume 2 of Int. Math. Ser. (N. Y.), pages 29-60. Kluwer/Plenum, New York, 2002.

[BBMN10] G. Bellettini, L. Bertini, M. Mariani, and M. Novaga. $\Gamma$-entropy cost for scalar conservation laws. Arch. Ration. Mech. Anal., 195(1):261-309, 2010.

[BI76] B. Bojarski and T. Iwaniec. Quasiconformal mappings and non-linear elliptic equations in two variables. i, ii. Bull. Acad. Polon. Sci. Sr. Sci. Math. Astronom. Phys., 22, 1976.

[BI82] B. Bojarski and T. Iwaniec. Another approach to liouville theorem. Math. Nachr., 107:253$262,1982$. 
[Boj74] B. Bojarski. Quasiconformal mappings and general structural properties of systems of non linear equations elliptic in the sense of Lavrentev, volume XVIII of Symposia Mathematica (Convegno sulle Transformazioni Quasiconformi e Questioni Connesse. INDAM, Rome, 1974.

[Bre11] H. Brezis. Functional analysis, Sobolev spaces and partial differential equations. New York, NY: Springer, 2011.

[CDL07] S. Conti and C. De Lellis. Sharp upper bounds for a variational problem with singular perturbation. Math. Ann., 338(1):119-146, 2007.

[CET94] P. Constantin, W. E, and E. S. Titi. Onsager's conjecture on the energy conservation for solutions of Euler's equation. Comm. Math. Phys., 165(1):207-209, 1994.

[DGF75] E. De Giorgi and T. Franzoni. Su un tipo di convergenza variazionale. Atti Accad. Naz. Lincei Rend. Cl. Sci. Fis. Mat. Natur. 5, 58:842-850, 1975.

[DiP85] R. J. DiPerna. Compensated compactness and general systems of conservation laws. Trans. Amer. Math. Soc, 292(2):383-420, 1985.

[DL89] R. J. DiPerna and P.-L. Lions. Ordinary differential equations, transport theory and Sobolev spaces. Invent. Math., 98(3):511-547, 1989.

[DLI15] C. De Lellis and R. Ignat. A regularizing property of the $2 D$-eikonal equation. Comm. Partial Differential Equations, 40(8):1543-1557, 2015.

[DLO03] C. De Lellis and F. Otto. Structure of entropy solutions to the eikonal equation. J. Eur. Math. Soc. (JEMS), 5(2):107-145, 2003.

[DMKO01] A. DeSimone, S. Müller, R. V. Kohn, and F. Otto. A compactness result in the gradient theory of phase transitions. Proc. Roy. Soc. Edinburgh Sect. A, 131(4):833-844, 2001.

[FJM02] G. Friesecke, R. D. James, and S. Müller. A theorem on geometric rigidity and the derivation of nonlinear plate theory from three-dimensional elasticity. Comm. Pure Appl. Math., 55(11), 2002.

[FK12] D. Faraco and J. Kristensen. Compactness versus regularity in the calculus of variations. Discrete Contin. Dyn. Syst. Ser. B, 17(2):473-485, 2012.

[FZ05] D. Faraco and X. Zhong. Geometric rigidity of conformal matrices. Ann. Sc. Norm. Super. Pisa Cl. Sci., 5(4):557-585, 2005.

[Geh62] F. W. Gehring. Rings and quasiconformal mappings in space. Trans. Amer. Math. Soc, 103(3):353-393, 1962.

[GL20] F. Ghiraldin and X. Lamy. Optimal besov differentiability for entropy solutions of the eikonal equation. Comm. Pure Appl. Math., 2020. to appear.

[Gra08] L. Grafakos. Classical Fourier analysis, volume 249 of Graduate Texts in Mathematics. Springer, New York, second edition, 2008.

[IM93] T. Iwaniec and G. Martin. Quasiregular mappings in even dimension. Acta Math., 170(1), 1993.

[IM12] R. Ignat and B. Merlet. Entropy method for line-energies. Calc. Var. Partial Differential Equations, 44(3-4):375-418, 2012.

[Iwa76] T. Iwaniec. Quasiconformal mapping problem for general nonlinear systems of partial differential equations. Symposia Mathematica, XVIII, 1976. 
[JK00] W. Jin and R. V. Kohn. Singular perturbation and the energy of folds. J. Nonlinear Sci., 10(3):355-390, 2000.

[JOP02] P.-E. Jabin, F. Otto, and B. Perthame. Line-energy Ginzburg-Landau models: zero-energy states. Ann. Sc. Norm. Super. Pisa Cl. Sci. (5), 1(1):187-202, 2002.

[Lio50] J. Liouville. Theoreme sur l'equation $d x^{2}+d y^{2}+d z^{2}=\lambda\left(d \alpha^{2}+d \beta^{2}+d \gamma^{2}\right)$. J. Math. Pures App., 1(15), 1850.

[LO18] X. Lamy and F. Otto. On the regularity of weak solutions to burgers' equation with finite entropy production. Calc. Var. Partial Differential Equations, 57(4), 2018.

[Lor14a] A. Lorent. Differential inclusions, non-absolutely convergent integrals and the first theorem of complex analysis. Q. J. Math., 65(4), 2014.

[Lor14b] A. Lorent. A quantitative characterisation of functions with low aviles giga energy on convex domains. Ann. Sc. Norm. Super. Pisa Cl. Sci., 13(5), 2014.

[LP18] A. Lorent and G. Peng. Regularity of the eikonal equation with two vanishing entropies. Ann. Inst. H. Poincaré Anal. Non Linéaire, 35(2):481-516, 2018.

[Mar] E. Marconi. On the structure of weak solutions to scalar conservation laws with finite entropy production. arXiv:1909.07257.

[MM77] L. Modica and S. Mortola. Un esempio di $\Gamma^{-}$-convergenza. Boll. Un. Mat. Ital. B (5), 14(1):285-299, 1977.

[MRv05] S. Müller, M. Rieger, and V. Šverák. Parabolic systems with nowhere smooth solutions. Arch. Ration. Mech. Anal., 177(1):1-20, 2005.

[Mv03] S. Müller and V. Šverák. Convex integration for lipschitz mappings and counterexamples to regularity. Ann. of Math., 9(3):715-742, 2003.

[MvY99] S. Müller, V. Šverák, and B. Yan. Sharp stability results for almost conformal maps in even dimension. J. Geom.Anal, 9(4), 1999.

[Pol07] A. Poliakovsky. Upper bounds for singular perturbation problems involving gradient fields. J. Eur. Math. Soc. (JEMS), 9(1):1-43, 2007.

[Res67] Yu. G Reshetnyak. Liouvilles conformal mapping theorem under minimal regularity hypotheses. (russian). Sibirsk. Mat. ̌̆, 8:835-840, 1967.

[RS01] T. Rivière and S. Serfaty. Limiting domain wall energy for a problem related to micromagnetics. Comm. Pure Appl. Math., 54(3):294-338, 2001.

[RS03] T. Rivière and S. Serfaty. Compactness, kinetic formulation, and entropies for a problem related to micromagnetics. Comm. Partial Differential Equations, 28(1-2):249-269, 2003.

[Sze04] L. Szekelyhidi, Jr. The regularity of critical points of polyconvex functionals. Arch. Ration. Mech. Anal., 172(1):133-152, 2004.

[Tri06] H. Triebel. Theory of function spaces. III, volume 100 of Monographs in Mathematics. Birkhäuser Verlag, Basel, 2006.

[Š93] V. Šverák. On Tartar's conjecture. Ann. Inst. H. Poincaré Anal. Non Linéaire, 10(4):405412, 1993.

[Zyg02] A. Zygmund. Trigonometric series. Vol. I, II. Cambridge Mathematical Library. Cambridge University Press, Cambridge, third edition, 2002. With a foreword by Robert A. Fefferman. 